\title{
Dynamic tuning of the director field in liquid crystal shells using block copolymers
}

\author{
JungHyun Noh, ${ }^{1}$ Yiwei Wang $\odot,{ }^{2}$ Hsin-Ling Liang, ${ }^{3}$ Venkata Subba Rao Jampani $\odot,{ }^{1}$ Apala Majumdar,,${ }^{4, *}$ \\ and Jan P. F. Lagerwall $\oplus^{1, \dagger}$ \\ ${ }^{1}$ Department of Physics and Materials Science, University of Luxembourg, Luxembourg City, Luxembourg \\ ${ }^{2}$ Department of Applied Mathematics, Illinois Institute of Technology, Chicago, Illinois 60616, USA \\ ${ }^{3}$ Institute of Organic Chemistry, Johannes Gutenberg University Mainz, Germany \\ ${ }^{4}$ Department of Mathematics and Statistics, University of Strathclyde, Glasgow, United Kingdom
}

(Received 2 April 2020; accepted 7 July 2020; published 28 July 2020)

\begin{abstract}
When an orientationally ordered system, like a nematic liquid crystal (LC), is confined on a self-closing spherical shell, topological constraints arise with intriguing consequences that depend critically on how the LC is aligned in the shell. We demonstrate reversible dynamic tuning of the alignment, and thereby the topology, of nematic LC shells stabilized by the nonionic amphiphilic block copolymer Pluronic F127. Deep in the nematic phase, the director (the average molecule orientation) is tangential to the interface, but upon approaching the temperature $T_{\mathrm{NI}}$ of the nematic-isotropic transition, the director realigns to normal. We link this to a delicate interplay between an interfacial tension that is nearly independent of director orientation, and the configuration-dependent elastic deformation energy of an LC confined in a shell. The process is primarily triggered by the heating-induced reduction of the nematic order parameter, hence realignment temperatures differ by several tens of degrees between LCs with high and low $T_{\mathrm{NI}}$, respectively. The temperature of realignment is always lower on the positive-curved shell outside than at the negative-curved inside, yielding a complex topological reconfiguration on heating. Complementing experimental investigations with mathematical modeling and computer simulations, we identify and investigate three different trajectories, distinguished by their configurations of topological defects in the initial tangential-aligned shell. Our results uncover a new aspect of the complex response of LCs to curved confinement, demonstrating that the order of the LC itself can influence the alignment and thereby the topology of the system. They also reveal the potential of amphiphilic block copolymer stabilizers for enabling continuous tunability of LC shell configuration, opening doors for in-depth studies of topological dynamics as well as novel applications in, e.g., sensing and programed soft actuators.
\end{abstract}

DOI: 10.1103/PhysRevResearch.2.033160

\section{INTRODUCTION}

Double emulsions of water in liquid crystal (LC) in water, also known as LC shells, have over the last decade acquired a status as a prolific experimental platform for studying confinement effects in soft matter physics [1-3], in particular concerning topological defects and their interactions on curved spaces. While the initial work, experimental and theoretical, was restricted to nematic shells (orientational order only; molecules aligning along the director n) [4-11], later efforts have focused also on smectic shells of SmA- [12-19] and SmC-type [17] as well as cholesteric [20-26] shells. Thanks to advances in providing long-term stability through polymerization/polymer stabilization of LC shells [26-29], they are also emerging as a realistic basis for innovative applications, for instance in photonics and photonics-derived use

\footnotetext{
*apala.majumdar@strath.ac.uk

$\dagger$ jan.lagerwall@lcsoftmatter.com
}

Published by the American Physical Society under the terms of the Creative Commons Attribution 4.0 International license. Further distribution of this work must maintain attribution to the author(s) and the published article's title, journal citation, and DOI. cases [20,26,27,30-35], sensing [28,36,37] or unconventional soft actuators [38-40].

For research as well as applications, it is imperative to control the director field configuration within the shell, a requirement typically fulfilled by choosing interface stabilizers that promote the desired orientation of $\mathbf{n}$ at each boundary $[41,42]$. Surprisingly little efforts have been devoted to exploring the vast parameter space of potential stabilizer molecules. One stabilizer, $\sim 85 \%$ hydrolyzed polyvinylalcohol (PVA), dominates entirely in work where tangential alignment (often also called planar alignment) is desired [5], and for normal (often called homeotropic) alignment the majority of studies use the anionic surfactant sodium dodecylsulfate (SDS) [7,42]. A recent systematic screening of cat- and anionic low molar mass surfactants revealed, however, that their impact is more subtle than expected [41]; depending on surfactant length and concentration, a fully tangential ("T"), a hybrid ("H," tangential on one side, normal on the other) or a fully normal ("N") configuration can be achieved. It is also possible that the stable alignment at a boundary is tilted, between tangential and normal, as has been demonstrated for lecithin-stabilized nematic droplets in glycerol [43]. Our understanding of how stabilizers affect the director field configuration in LC shells is thus far from complete. 
Even with well-defined uniform boundary conditions, there may be significant variability in the director field and distribution of topological defects in the shell. This is particularly the case for shells in a $\mathrm{T}$ configuration, a situation that has been extensively investigated, experimentally $[5,9,13,14,29,44]$ as well as theoretically and by computer simulation $[4,6,8-$ 11,44-46]. The Poincaré-Hopf theorem dictates that the inside as well as the outside of a $\mathrm{T}$ shell must have a total topological defect charge of $+2[1,3]$. This requirement can be fulfilled in three ways, all of which have been found experimentally and in simulation.

(1) Four $+1 / 2$ disclination lines connect the shell inside and outside. We will refer to this tangential configuration, which is the most common, as T1, alternatively the more descriptive label $4\left(+\frac{1}{2}\right)$.

(2) Two $+1 / 2$ disclination lines connect the shell inside and outside, the remaining +1 topological charge at each interface being realized by a point defect. We call this configuration $\mathrm{T} 2$, with the descriptive label $2\left(+\frac{1}{2}\right),+1$.

(3) The inside as well as the outside each have two +1 point defects. We label this tangential configuration T3, alternatively $2(+1)$.

The $\mathrm{H}$ configuration has so far been investigated in detail only experimentally $[15,17,42]$, revealing that the observable stable hybrid configuration always has two antipodal +1 point defects on the tangentially aligned side of the shell, one at the thinnest and one at the thickest point. A shell in the $\mathrm{N}$ configuration is defect-free, as its radial director field has only a virtual bulk defect at the center of the shell, which is occupied by the isotropic liquid core.

While most LC shells have permanent configuration once produced, it is very powerful to be able to dynamically tune the director field after production, for scientific experiments and applications. Such realignment has been achieved twice for shells, first via an exchange of stabilizer in the outer phase [7], then using a custom-synthesized light-switchable additive [47]. For droplets, Volovik and Lavrentovich achieved realignment of the single outer interface simply by heating lecithinstabilized nematic droplets dispersed in glycerol throughout the nematic temperature range [43]. Deep in the nematic phase, the droplets were normally aligned, while they adopted tangential alignment near the clearing point. The change was mediated via a continuous increase of director tilt with respect to the interface upon heating.

Here we demonstrate that temperature-driven reversible dynamic tuning of the configuration is possible also with nematic shells in water, using the commercially available amphiphilic block copolymer Pluronic F127 [Fig. 1(a)] for stabilizing the interfaces. F127 consists of two hydrophilic polyethyleneoxide (PEO) blocks separated by a hydrophobic polypropyleneoxide (PPO) block, with the overall composition $\mathrm{PEO}_{99}-\mathrm{PPO}_{67}-\mathrm{PEO}_{99}$. In preliminary experiments [12,17] we noticed the remarkable ability of F127-stabilized shells to change alignment with temperature $T$ : at room temperature the shells are in a $\mathrm{T}$ configuration, but on heating they switch first to $\mathrm{H}$ and then to $\mathrm{N}$ configuration near the nematicisotropic transition (clearing) at $T=T_{\mathrm{NI}}$. These early studies were carried out on shells of 4-Octyl-4'-cyanobiphenyl (8CB), with a phase sequence Cr. $21.5^{\circ} \mathrm{C} \mathrm{SmA} 33^{\circ} \mathrm{C} \mathrm{N}$ $40.5^{\circ} \mathrm{C}$ iso. We thus speculated that the configuration changes

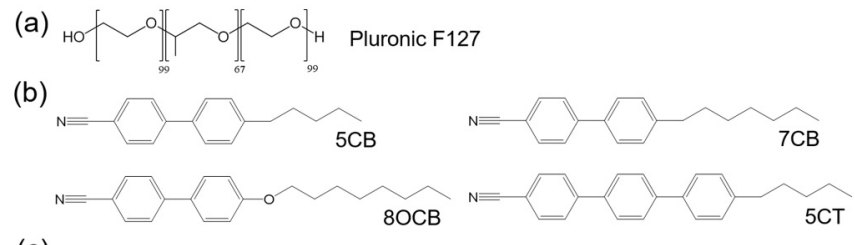

(c)

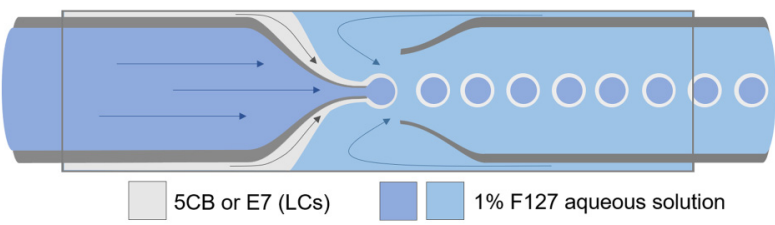

FIG. 1. Molecular structures of (a) Pluronic F127 block copolymer and (b) mesogens; $5 \mathrm{CB}, 7 \mathrm{CB}, 8 \mathrm{OCB}$ and $5 \mathrm{CT}$. The mixture of the four components yields E7 (mixing ratio in Sec. II A). (c) Schematic illustration of the production of LC shells using a coaxial microfluidic device.

may have been related to the development/disappearance of smectic order.

In the current study we refute this initial hypothesis, by presenting detailed investigations of shells of two LC materials [Fig. 1(b)] without smectic phases, 4-cyano4'-pentyl biphenyl (5CB) and the mixture E7. We find the same sequence of reversible configuration changes, $\mathrm{T}_{\text {heat }} \overrightarrow{\mathrm{H}_{\text {heat }}} \underset{\mathrm{N}}{\overrightarrow{\mathrm{Nool}}} \overrightarrow{\mathrm{H}_{\text {cool }}} \rightarrow$, on heating and subsequent cooling, showing that smectic order is not required. It is primarily the variation of orientational order with temperature that drives the changes. Significantly, the temperatures at which configurational changes take place are very different for the two LCs, a consequence of the higher clearing point of E7 $\left(T_{\mathrm{NI}} \approx 59^{\circ} \mathrm{C}\right)$ than of $5 \mathrm{CB}\left(T_{\mathrm{NI}}=35.5^{\circ} \mathrm{C}\right)$. We complement the experiments with theoretical modeling and numerical results, providing new insights into nematic equilibria in hybrid shells, twisted director profiles and dynamical pathways from tangential to hybrid, that mimic experiments.

\section{EXPERIMENT AND SIMULATION DETAILS}

\section{A. Experimental shell production and characterization}

LC shells are fabricated using a glass capillary-based microfluidic emulsification device, see Fig. 1(c), all capillaries from WPI Instruments. Two cylindrical capillaries $(1 \mathrm{~mm}$ outer diameter) are coaxially fitted into a square capillary (inner dimension $1 \mathrm{~mm}$ ), the former tapered at one end and carefully aligned with nozzles facing each other. These serve as injection and collection tube, respectively. Unless otherwise stated, a $1 \mathrm{wt} . \%$ aqueous solution of F127 serves as inner as well as outer phase. The inner phase is pumped through the injection tube with the LC forming the middle fluid, flowing in the same direction through the voids between injection tube and square capillary. It thus coats the inner fluid at the orifice. The outer phase is flown in the opposite direction through the voids between collection tube and square capillary, flow focusing the LC-inner phase coflow into the collection tube, where the compound jet breaks into discrete LC shells. The injection tube is hydrophobically treated to prevent wetting by the inner aqueous phase at the tip region. A temperature 
controlled heating cavity houses the device, so LC shells can be produced in the isotropic phase where the flow is the easiest. The diameter of the produced shells is in the range $100-120 \mu \mathrm{m}$ and the average thickness 3-6 $\mu \mathrm{m}$.

\section{Liquid crystals}

We make shells from 5CB (Cr. $24 \mathrm{~N} 35.5$ Iso. $/{ }^{\circ} \mathrm{C}$; the nematic phase is easily supercooled to $\sim 20^{\circ} \mathrm{C}$ ) as well as from the four-component mixture E7 (nematic on cooling to $-60^{\circ} \mathrm{C}$, clearing range on heating about $58.5^{\circ} \mathrm{C}-60^{\circ} \mathrm{C}$ ), composed of the four cyanobiphenyl-based mesogens $5 \mathrm{CB}$ (47 mole\%), 7CB (4-cyano-4'-heptyl biphenyl, 25 mole\%), 8OCB (4-cyano-4'-octyloxy biphenyl, 18 mole\%) and 5CT (4-cyano-4'-pentyl terphenyl, 10 mole\%). All structures are shown in Fig. 1.

\section{POM and FCPM characterization}

Shell suspensions are filled into rectangular capillaries (200 $\mu \mathrm{m}$ thickness) and observed in a polarizing optical microscope (POM, Olympus BX51) equipped with a hot stage. For fluorescence confocal polarization microscopy (FCPM, Nikon A1R+) to map out the LC director field $[47,48]$ a small amount $(0.1 \%)$ of dichroic dye (BTBP: N,N'-bis(2,5-di-tertbutylphenyl)- 3,4,9,10-perylene-carboximide) is mixed into the LC before shell production. A capillary holding the shells is placed on a home-built resistive heating device, consisting of a $1 \mathrm{~mm}$ thick glass plate coated with transparent ITO (indium tin oxide) electrodes connected to a temperatureregulated power supply. The BTBP dye is excited by $488 \mathrm{~nm}$ laser light and the fluorescence emission is collected at 525 $\mathrm{nm}$. The data are visualized in 3D using Nikon NIS elements imaging software.

\section{B. Theoretical and numerical methods}

In this section, we summarize our theoretical methods for simulating experimentally observable equilibrium nematic textures and their transient dynamics inside asymmetric shells. The shell is defined by

$$
\Omega=B(\mathbf{0}, R) \backslash B\left(\boldsymbol{x}_{c}, R_{1}\right) \subset \mathbb{R}^{3},
$$

where $B\left(\boldsymbol{x}_{c}, R_{1}\right) \subset B(\mathbf{0}, R) . \quad B(\mathbf{0}, R)$ denotes a threedimensional ball of arbitrary radius $R$ centered at the origin, $B\left(x_{c}, R_{1}\right)$ is a smaller ball of radius $R_{1}$ centered at the point $\boldsymbol{x}_{c}=(0,0, c R) \in B(0,1)$ for $c<1$. The shell has two boundaries, the outer boundary is $\partial B(\mathbf{0}, R)$ whereas the inner boundary is $\partial B\left(x_{c}, R_{1}\right)$. In what follows, we model shells with preferred tangential anchoring on $\partial B\left(x_{c}, R_{1}\right)$ and with either tangential anchoring on $\partial B(\mathbf{0}, R)$ (referred to as fully tangential shells) or with normal anchoring on $\partial B(\mathbf{0}, R)$ (referred to as hybrid shells).

We work within the powerful continuum Landau-de Gennes (LdG) theory for nematic LCs. The LdG theory describes the nematic phase by a macroscopic LdG Q-tensor parameter, which is a symmetric traceless $3 \times 3$ matrix that encodes information about the state of orientational order [49,50]. The phase is said to be isotropic if $\mathbf{Q}=0$ with no orientational ordering, uniaxial nematic if $\mathbf{Q}$ has a single distinguished eigendirection, the usual director $\mathbf{n}$, and biaxial nematic if $\mathbf{Q}$ has three distinct eigenvalues, rendering a primary and secondary director. A uniaxial Q-tensor can be written as

$$
\mathbf{Q}=s\left(\mathbf{n} \otimes \mathbf{n}-\frac{\mathbf{I}}{3}\right)
$$

where $s$ is a scalar order parameter that measures the degree of ordering about $\mathbf{n}$. A further metric of orientational ordering is the biaxiality parameter

$$
\beta=1-6 \frac{\left(\operatorname{tr} \mathbf{Q}^{3}\right)^{2}}{\left(\operatorname{tr} \mathbf{Q}^{2}\right)^{3}} .
$$

One can show $0<\beta \leqslant 1$ in the biaxial region, while $\beta=0$ in the uniaxial region $[49,51]$. Biaxiality is particularly important for visualizing and understanding the structure of nematic defect cores.

The LdG theory is a variational theory so that the experimentally observable configurations are modeled by either local or global minimizers of an appropriately defined LdG free energy [49,52]. We work with the following well accepted form of the $\mathrm{LdG}$ free energy:

$$
\mathcal{F}[\mathbf{Q}]=\int_{\Omega} f_{\mathrm{b}}(\mathbf{Q})+f_{\mathrm{el}}(\mathbf{Q}, \nabla \mathbf{Q}) d V+\int_{\partial \Omega} f_{\mathrm{s}}(\mathbf{Q}) d S,
$$

where $f_{\mathrm{b}}$ is the bulk energy density, $f_{\mathrm{el}}$ is the elastic energy density, and $f_{\mathrm{s}}$ is the surface energy density. The bulk energy density, $f_{\mathrm{b}}$, is given by

$$
f_{\mathrm{b}}(\mathbf{Q})=\frac{A}{2} \operatorname{tr}\left(\mathbf{Q}^{2}\right)-\frac{B}{3} \operatorname{tr}\left(\mathbf{Q}^{3}\right)+\frac{C}{4}\left(\operatorname{tr}\left(\mathbf{Q}^{2}\right)\right)^{2},
$$

where $A=a\left(T-T_{\mathrm{NI}}^{*}\right)$, with $T_{\mathrm{NI}}^{*}$ the nematic supercooling limit such that the isotropic phase is unstable for $T<T_{\mathrm{NI}}^{*}$, and $a, B$ and $C$ are material dependent parameters. We can explicitly compute the minimizers of $f_{\mathrm{b}}$. For $A<\frac{B^{2}}{27 C}$, $f_{\mathrm{b}}$ attains its minimum on the set of uniaxial $\mathbf{Q}$ tensors defined by

$$
\mathcal{N}=\left\{\mathbf{Q}=s^{+}\left(\mathbf{m} \otimes \mathbf{m}-\frac{\mathbf{I}}{3}\right)\right\},
$$

where $s^{+}=\frac{B+\sqrt{B^{2}-24 A C}}{4 C}$ is referred to as the "optimal" scalar order parameter and $\mathbf{m}$ is an arbitrary unit-vector field. For a given set of material constants, the isotropic-nematic phase transition takes place at $A=\frac{B^{2}}{27 C}[49,53]$. The elastic energy density is often given by

$$
\begin{aligned}
f_{\mathrm{el}}(\mathbf{Q}, \nabla \mathbf{Q})= & \frac{L_{1}}{2} Q_{i j, k} Q_{i j, k}+\frac{L_{2}}{2} Q_{i j, j} Q_{i k, k} \\
& +\frac{L_{3}}{2} Q_{i k, j} Q_{i j, k}+\frac{L_{4}}{2} Q_{k l} Q_{i j, k} Q_{i j, l},
\end{aligned}
$$

where the $L_{i}(i=1 \ldots 4)$ are experimentally measurable material elastic constants [54]. For relative simplicity and wellposedness, we take $L_{3}=L_{4}=0$ in what follows (see Ref. [54] for problems with $L_{4} \neq 0$ ). The elastic constants can be correlated to certain specific material deformations, e.g., splay, twist or bend. This can be more clearly seen if we compare $f_{\mathrm{el}}$ with the Oseen-Frank (OF) free energy density for uniaxial 
nematics, with $\mathbf{Q}=s\left(\mathbf{n} \otimes \mathbf{n}-\frac{\mathbf{I}}{3}\right)$ and constant $s$ [53]:

$$
\begin{aligned}
W[\mathbf{n}]= & K_{1}(\nabla \cdot \mathbf{n})^{2}+K_{2}(\mathbf{n} \cdot(\nabla \times \mathbf{n}))^{2}+K_{3}|\mathbf{n} \times(\nabla \times \mathbf{n})|^{2} \\
& +\left(K_{2}+K_{4}\right)\left(\operatorname{tr}(\nabla \mathbf{n})^{2}-(\nabla \cdot \mathbf{n})^{2}\right) .
\end{aligned}
$$

Here, $K_{1}, K_{2}$, and $K_{3}$ are the elastic constants that describe the energetic penalty of splay, twist, and bend deformations, respectively. $K_{4}$ is a saddle-splay elastic constant usually associated with surface effects; it does not contribute to the Oseen-Frank energy of tangentially aligned nematic droplets and we do not include it in this manuscript [49,55]. Tran et al. compared simulations with and without saddle-splay constants for long-pitch cholesteric shells [23], concluding that the approximation $K_{4}=0$ introduces no significant errors.

While the OF theory is not as general as the LdG theory, we can make a correspondence between the elastic constants in the LdG theory and the OF theory as shown below, with $L_{3}=L_{4}=0$ [53].

$$
\begin{array}{ll}
K_{1}=\left(2 L_{1}+L_{2}\right) s^{2}, & K_{2}=2 L_{1} s^{2}, \\
K_{3}=\left(2 L_{1}+L_{2}\right) s^{2}, & K_{4}=0,
\end{array}
$$

which implies $K_{2}<K_{1}=K_{3}$ for $L_{2}>0$ and

$$
\frac{K_{1}}{K_{2}}=1+\frac{1}{2} \frac{L_{2}}{L_{1}} \text {. }
$$

We define $\eta=L_{2} / L_{1}$ so that $K_{1} / K_{2}=1+\eta / 2$. In particular, increasing $\eta$ is equivalent to an increased energetic penalty for splay deformations, so that twisted configurations are more readily observable for large and positive $\eta$ (see Ref. [55] for formal calculations of the energies of twisted configurations on tangentially aligned nematic droplets). The case $\eta=0$ describes the one-constant approximation with $K_{1}=K_{2}=K_{3}$ and $K_{4}=0$ [49].

A further key modeling ingredient are the boundary conditions. In the case of hybrid shells, we impose normal anchoring on the outer shell by means of a Rapini-Papoular energy [56]

$$
F_{\mathrm{s}}=\frac{W_{0}}{2} \int_{\partial B(\mathbf{0}, R)}\left(Q_{i j}(\mathbf{x})-Q_{i j}^{\mathrm{s}}(\mathbf{x})\right)^{2} d S,
$$

where $W_{0}$ is the anchoring strength,

$$
\mathbf{Q}^{\mathrm{s}}(\boldsymbol{x})=s^{+}\left(\boldsymbol{v}(\boldsymbol{x}) \otimes \boldsymbol{v}(\boldsymbol{x})-\frac{1}{3} \mathbf{I}\right), \quad \boldsymbol{x} \in \partial \Omega,
$$

and $\boldsymbol{v}$ is the surface normal of $\partial B(0, R)$. In the limit of $W_{0} \rightarrow+\infty$, we recover strong normal anchoring since $F_{\mathrm{s}}$ is minimized by $\mathbf{Q}=\mathbf{Q}^{\mathrm{s}}$ for $W_{0}>0$. We do not consider $W_{0}<0$ in this manuscript but $W_{0}<0$ favors boundary alignments that are "far" away from the idealized normal anchoring $\mathbf{Q}^{\text {s }}$; this could be tangential anchoring, tilted anchoring etc.

The tangential degenerate anchoring is imposed on the inner surface, $\partial B\left(\boldsymbol{x}_{c}, R_{1}\right)$ by the following surface energy [57]:

$$
F_{S}=\int_{\partial B\left(x_{c}, R_{1}\right)} \frac{W_{1}}{2}\left(\widetilde{Q}_{i j}-\widetilde{Q}_{i j}^{\perp}\right)^{2} d S,
$$

where $\widetilde{Q}_{i j}=Q_{i j}+\frac{1}{3} s^{+} \delta_{i j}$,

$$
\widetilde{Q}_{i j}^{\perp}=P_{i k} \widetilde{Q}_{k l} P_{l j}, \quad \mathbf{P}=\mathbf{I}-\boldsymbol{v} \otimes \boldsymbol{v} .
$$

The surface energy in (9) favors the tangential projection of $\mathbf{Q}$ onto the surface of $\partial B\left(\boldsymbol{x}_{c}, R_{1}\right)$ without imposing specific directors i.e. it is minimized for $\widetilde{Q}_{i j}=\widetilde{Q}_{i j}^{\perp}$ for $W_{1}>0$ and $\widetilde{Q}_{i j}^{\perp}$ is the tangential projection of $\widetilde{Q}$ on $\partial B\left(x_{c}, R_{1}\right)$. We note that $F_{s}$ in (9) could include tilted anchoring for moderate values of $W_{1}$ but we do not consider intermediate values in this manuscript.

It is important to nondimensionalize the LdG free energy, so that we can study the dependence of the equilibria on key material characteristics, e.g., elastic anisotropy, relative temperature, anchoring and geometrical size. We nondimensionalize the free-energy (1) by using $R$ as the characteristic length. The re-scaled domain becomes $\Omega=D_{0} \backslash D_{1}$,

$$
D_{0}=B(\mathbf{0}, 1), \quad D_{1}=B\left(\boldsymbol{x}_{c}, \rho\right),
$$

where $\rho=R_{1} / R, \boldsymbol{x}_{c}=(0,0, c)$ as before. The parameters $c$ and $\rho$ satisfy $c \geqslant 0$ and $c+\rho<1$. These parameters control the asymmetry and thickness of the shell. For example, $c=$ 0 describes a symmetric shell whereas $c>0$ describes a shell that is thinner towards the top. Smaller values of $c+\rho$ describe thicker shells since the shell thickness is proportional to the quantity, $1-c-\rho$. Hence, the rescaled boundary is $: \partial \Omega=\partial D_{0} \cup \partial D_{1}$. Let

$$
\overline{\boldsymbol{x}}=\boldsymbol{x} / R, \quad \overline{\mathbf{Q}}=\sqrt{\frac{27 C^{2}}{2 B^{2}}} \mathbf{Q}, \quad \overline{\mathcal{F}}=\frac{27^{2} C^{3}}{2 B^{4} R^{3}} \mathcal{F},
$$

Dropping all bars for convenience, the dimensionless LdG free energy for a hybrid shell is given by

$$
\begin{aligned}
\mathcal{F}[\mathbf{Q}]= & \int_{\Omega} \frac{t}{2} \operatorname{tr}\left(\mathbf{Q}^{2}\right)-\sqrt{6} \operatorname{tr}\left(\mathbf{Q}^{3}\right)+\frac{1}{2}\left(\operatorname{tr}\left(\mathbf{Q}^{2}\right)\right)^{2} \\
& +\frac{\xi_{R}^{2}}{2}\left(Q_{i j, k} Q_{i j, k}+\eta Q_{i j, j} Q_{i k, k}\right) d \mathbf{x} \\
& +\int_{D_{1}} \frac{w_{0}}{2}\left(Q_{i j}(\mathbf{x})-Q_{i j}^{\mathrm{s}}(\mathbf{x})\right)^{2} d A \\
& +\int_{D_{2}} \frac{w_{1}}{2}\left(\widetilde{Q}_{i j}-\widetilde{Q}_{i j}^{\perp}\right)^{2} d A,
\end{aligned}
$$

where $t=\frac{27 A C}{B^{2}}$ is a dimensionless temperature, $\xi_{R}=\sqrt{\frac{27 C L_{1}}{B^{2} R^{2}}}$ is related to the shell size, $\eta=\frac{L_{2}}{L_{1}}\left(K_{1} / K_{2}=1+\frac{1}{2} \eta\right)$ is a measure of the elastic anisotropy. The nondimensionalized anchoring strength is given by $w_{i}=\frac{27 C W_{i}}{B^{2} R}(i=0,1)$. In the simulation, we adopt the parameter values $A=-0.172 \times$ $10^{6} \mathrm{~J} \mathrm{~m}^{-3}, B=-2.12 \times 10^{6} \mathrm{~J} \mathrm{~m}^{-3}, C=1.73 \times 10^{6} \mathrm{~J} \mathrm{~m}^{-3}$, and $L_{1}=4 \times 10^{-11} \mathrm{~J} \mathrm{~m}^{-1}$, which are typical phenomenological values for $5 \mathrm{CB}$ at room temperature [58,59]. This motivates us to use $t=-1.79$ for most of the simulations, since $t=-1.79$ corresponds to the room temperature for $5 \mathrm{CB}$, while $t=1$ is the nematic-isotropic transition temperature. The anchoring strength $W_{0}$ and $W_{1}$ are taken to be $10^{-2} \mathrm{Jm}^{-2}$ to account for the strong anchoring [59], if not stated differently.

The details of the numerical methods can be found in Appendix B. We use the spectral method to discretize the tensor order parameter $\mathbf{Q}$, that is expanding $\mathbf{Q}$ in terms of proper basis functions after the appropriate identification of a coordinate system [60]. Neglecting the high-order terms in a truncated series, we express the free energy in terms of undetermined coefficients and use standard optimization methods, 

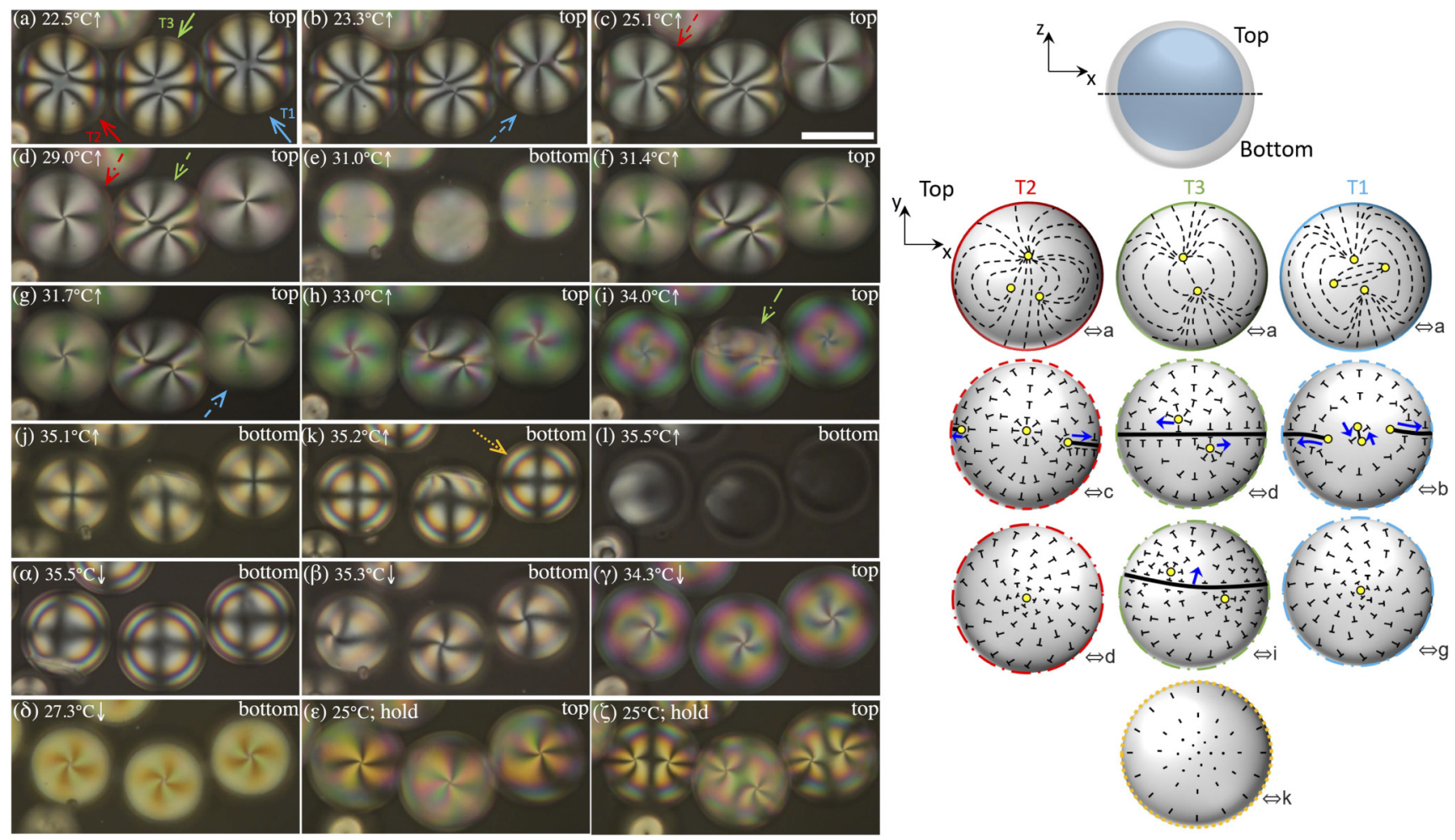

FIG. 2. Freshly produced 5CB shells heated from room temperature to isotropic [(a)-(1)] and then cooled back $[(\alpha)-(\epsilon)]$ at moderate cooling rate, holding at $25^{\circ} \mathrm{C}$ at the end $[(\epsilon)-(\zeta)]$. Initially (a), the three shells have one each of the possible tangential configurations: T1 on the right, T2 on the left, and T3 in the middle. However, after the cooling experiment, all shells end up as T3. The shells are viewed along gravity, the texts "top" and "bottom" indicating the focal plane. Near room temperature they appear elliptical due to an optical artifact that is explained in Appendix A. Scale bar: $100 \mu \mathrm{m}$. On the top right, a sketch of a shell from the side is drawn to illustrate the asymmetry, with thin top and thick bottom. No photo corresponds to this perspective. Below are sketches drawn in the same perspective as the photos, showing the top of each shell as viewed along gravity, of the director field for representative steps in the heating sequence. The color and character of the ring around each drawing matches those of the arrow pointing to the corresponding shell photo on the left. Localized defects are drawn in yellow and defect lines (explained in Sec. III B) as black lines. The blue arrows indicate direction of motion of a defect or line.

to minimize the discrete free energy and compute local or global energy minimizers. For the dynamical transitions, we solve a gradient flow system for $\mathbf{Q}$, which are based on the principle that the free energy decreases with time till we settle into a local energy minimum.

Our numerics are limited to shells with diameter on the micron scale $(1-5 \mu \mathrm{m})$, similar to other theoretical studies $[61,62]$ and are hence two orders of magnitude smaller than the shells in experiments (100-150 $\mu \mathrm{m}$ diameter). In some situations, this may lead to different results.

\section{RESULTS}

\section{A. 5CB shell textures on heating/cooling}

\section{Experimental observations}

We heat $5 \mathrm{CB}$ shells surrounded by $1 \%$ aqueous solutions of F127 from room temperature to slightly above $T_{\mathrm{NI}}=35.5^{\circ} \mathrm{C}$ and then cool back. This experiment, as observed through the POM, is shown in movie 1 in Ref. [63], with representative snapshots on three adjacent shells in Fig. 2. The shells start out pristine, i.e., they have gone through no temperature changes after the rapid cooling from isotropic to nematic at the end of the production process. The director configurations at room temperature [Fig. 2(a)] correspond to tangential boundary condition on in- and outside ( $T$ configuration). The three selected shells represent all possible combinations of topological defects in a T configuration, as defined above.

(1) The T1 configuration $\left[4\left(+1 / 2^{t}\right)\right]$ is seen in the right shell, highlighted by a blue arrow and labeled T1 in Fig. 2(a).

(2) The T2 configuration $\left[2\left(+1 / 2^{t}\right),+1^{t}\right]$ is seen in the left shell (red arrow), labeled T2 in Fig. 2(a).

(3) The T3 configuration $\left[2\left(+1^{t}\right)\right]$ appears in the middle shell (green arrow), labeled T3 in Fig. 2(a).

In the descriptive configuration notation, we have here added lower-case superscripts to indicate where a defect is located: " $t$ " stands for the "top" of the shell. The top is also the thinnest point, as the aqueous F127 solution is less dense than the LC.

In total we have studied hundreds of shells similar to those in Fig. 2. In freshly produced shells at room temperature we always find all three configurations, with a strong preference for $\mathrm{T} 1\left[4\left(+1 / 2^{t}\right)\right]$. In an experiment with a total of 55 pristine shells we counted 40 in T1, 9 in T2, and 6 in T3 configuration.

As we heat and then cool the shells, we identify several surprising features in the textural evolution, with some differences depending on shell type. We briefly go through the key observations here, in the order of the starting configurations, 
T1-T3. This means that we will discuss first the right shell (blue arrows), then the left shell (red arrows), and finally the middle shell (green arrows) in Fig. 2. In Sec. III B, we go through each trajectory in more detail, based on the expanded temperature range observations in E7 shells.

(1) In all cases, a shell changes gradually on heating from the original $\mathrm{T}$ configuration, via $\mathrm{H}$, and finally to $\mathrm{N}$ [Figs. 2(a)-2(n)]. During the $\mathrm{T}_{\text {heat }} \mathrm{H}$ transition we can distinguish two or three characteristic steps, depending on the starting configuration.

(a) Starting with the T1 shell $\left[4\left(+1 / 2^{t}\right)\right.$, blue arrow], two of the four $+1 / 2$ defects first move to the top [Figs. 2(b) and 2 (c)] and remain there. They are very close but do not yet fuse. We define this as step 1 of the T1-initiated trajectory. The other two defects first move apart towards the equator [Figs. 2(b)-2(d)], then approach each other on the bottom side of the shell (e). Between Figs. 2(f) and 2(g) the two top $+1 / 2$ defects fuse into a single +1 defect, with the sudden appearance of a spiral pattern in POM signifying a twist in the director field in the top half of the shell. We define this as step 2 , occurring before the remaining $+1 / 2$ defects have met at the bottom. They do so in step 3 [just before Fig. 2(i), see movie 1], immediately fusing and leaving a second +1 point defect antipodal to the first +1 defect. Again, the defect fusion is connected to the sudden appearance of a spiral pattern, this time on the bottom shell half, hence the director field twist now extends throughout the shell. The end configuration is thus a hybrid $+1^{b},+1^{t}$ (superscript " $b$ " for "bottom").

The three steps of this trajectory are illustrated graphically from multiple perspectives in Fig. 3. It is here easy to see that Step 1 induces no twist [(a)-(c)], whereas between step 2 and step 3 [(d)-(f)] a twist is localized to the top half of the shell. After step 3, finally [(g)-(i)], the director field twists throughout the shell. While the drawing is done for one twisting sense, the sense in experiments varies randomly between shells, as expected without molecular chirality.

(b) Next, we study the closely related trajectory starting from T2 $\left[2\left(+\frac{1}{2^{t}}\right),+1^{t}\right]$. This trajectory exhibits the same final steps as defined above, but step 1 is absent, since only two $+1 / 2$ defects are present from the beginning. As we heat from room temperature, the initial +1 defect moves up to the top of the shell, where it stops [Fig. 2(c)]. Soon afterwards we see the characteristic spiral pattern revealing a director twist in the top shell half, see Fig. 2(d), constituting step 2. As above, the two $+1 / 2$ defects first move apart towards the equator, which they reach at about the temperature of step 2 . On continued heating, they then approach each other on the bottom side of the shell, where they eventually fuse into a +1 point defect (step 3 ) that is antipodal to the original one, see Fig. 2(f). Again, step 3 coincides with the sudden appearance of a spiral pattern on the bottom half of the shell, showing that the director field twist now extends throughout the shell. The stable hybrid configuration thus again ends up $+1^{b},+1^{t}$.

(c) The T3 shell $\left[2\left(+1^{t}\right)\right]$, finally, gives rise to a realignment sequence that is altogether different from the two trajectories described above. We identify three key steps here, allowing us to compare the temperatures at which these steps occur in 5CB and E7 shells, but we note that they are not necessarily directly comparable to the steps seen in the trajectories starting from $\mathrm{T} 1$ or $\mathrm{T} 2$. In the study of the
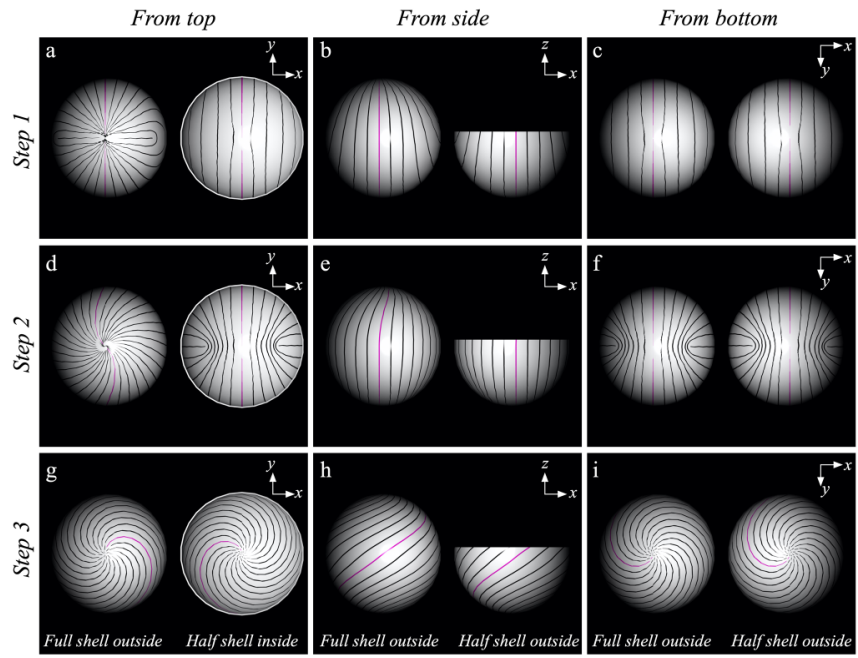

FIG. 3. Computer-drawn illustrations of how the tangential director field projection changes in shells following the three-step realignment trajectory that begins with the $\mathrm{T} 1\left[4\left(+1 / 2^{t}\right)\right]$ configuration. In each panel, one full shell (left) and one half shell (right) is drawn, both with identical tangential director fields mapped onto them. One field line has been highlighted in pink color, to facilitate tracing the director around the shell surface. The shells are viewed along three perspectives, from the top in the left column, thus looking into the half shell, from the side in the middle column, and from the bottom in the right column, viewing both shells from their outsides. The drawings correspond to the (predominantly) tangential shell side immediately following step 1 [(a)-(c)], step 2 [(d)-(f)], and step $3[(\mathrm{~g})-(\mathrm{i})]$, respectively. The perspective in the left-most column corresponds to that in upright microscopy, the full shell showing the texture obtained with focus on the top and the half shell the texture when the focus is at the bottom. In (a), the two $+1 / 2$ defects at the shell top, gathered very close without merging into $a+1$ defect, cannot be well resolved with the graphics software used.

$\mathrm{T} \rightarrow \mathrm{H}$ transition upon stabilizer exchange by Lopez-Leon and Fernandez-Nieves [7], the starting configuration was also $2\left(+1^{t}\right)$, and the trajectory we observe in the T3 shell matches one of the trajectories described in their report. A defect ring first arises along a great circle between the two +1 point defects, which soon start leaving the top, moving towards the equator along the ring in opposite directions [Figs. 2(c) and $2(\mathrm{~d})]$. In this trajectory, we call the start of the defect movement step 1. Somewhat later, the ring leaves the great circle location, moving towards one side and shrinking in the process [Fig. 2(i)]. Initially both point defects move with the ring, but, as seen in movie 1, soon one defect detaches (we denote this step 2 of this trajectory), moving up to the top of the shell. In the experiment of Fig. 2 and movie 1, the transition to fully normal alignment (see below) occurs in the T3-initiated shell prior to completion of the $\mathrm{T}_{\text {heat }} \mathrm{H}$ transition by step 3 , hence we will come back to the final stage in Sec. III B 3. The extended temperature range of E7 shells there allows us to follow the $\mathrm{T}_{\text {heat }} \mathrm{H}$ transition until the end.

(2) On yet further heating, the shell turns from hybrid to fully normal $\left(\mathrm{H}_{\text {heat }} \mathrm{N}\right)$, see Figs. 2(j) and 2(k). This happens less than $1 \mathrm{~K}$ below $T_{\mathrm{NI}}$. Continuing to heat to $T_{\mathrm{NI}}$, the shell turns isotropic [Fig. 2(1)]. 

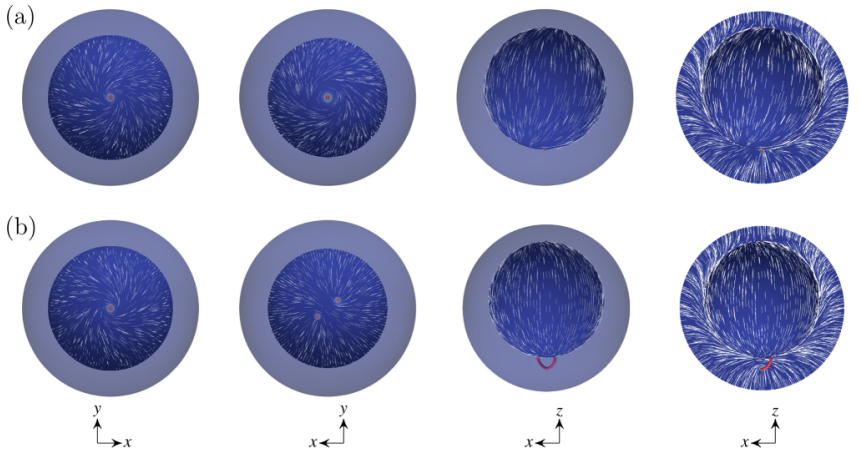

FIG. 4. Two (meta-) stable $\mathrm{H}$ states (tangential inside, normal outside) obtained from numerical simulations, with the reduced temperature (different from the experimentally defined $T_{r}$, used below) $t=-1.79, \quad c=0.1, \rho=0.7$, and $\eta=4$. The shells are viewed from the thinnest part (shell top), thickest part (bottom), and side, respectively, from left to right. The final two columns have the same side view perspective, the third column showing the director field only at the inner shell boundary while the fourth column shows the director field within the shell. With two antipodal +1 defects $\left(\mathrm{a} ;+1^{b},+1^{t}\right.$ configuration) the director field is twisted from top to bottom, whereas a shell with a $2\left(+^{1} 2^{b}\right),+1^{t}$ configuration (b) exhibits twist only on the top half, where the +1 defect resides. Both $+1 / 2$ defects at the bottom are on the inside, connected by a U-turned disclination line. Calculations of the LdG free energy show that $+1^{b},+1^{t}$ is the global minimum whereas $2\left(+\frac{1 / 2^{b}}{)},+1^{t}\right.$ is a local minimum.

(3) Cooling the shells back to nematic, the alignment transitions take place in reverse: $\mathrm{N}_{\text {cool }} \mathrm{H}$ with twisted director field [Figs. $2(\alpha)-2(\gamma)$ ], finally $\mathrm{T}$ after a few minutes at room temperature [Figs. $2(\delta)-2(\zeta)]$.

(4) Significantly, the final T state [Fig. $2(\zeta)]$ shows only one type of tangential director field: T3 $\left[2\left(+1^{t}\right)\right]$. No single shell displays any $+1 / 2$ disclination after the slow cooling process of [Figs. $2(\alpha)-2(\gamma)$ ], thus T1 and T2 configurations are entirely absent. In the quantitative experiment mentioned above, all 55 shells remained intact after the slow cooling and all were in T3 configuration. We will propose an explanation for this observation, which we believe is intimately linked to the intermediate $\mathrm{H}$ configuration upon slow cooling, in Sec. IV C.

\section{Simulation of stable hybrid configurations}

We first focus on the stable H configuration, see Fig. 4, setting the inside boundary condition tangential and the outside normal; this is compatible with experimental data above and identical to the starting configuration in Sec. III C. To compare our numerical results with earlier studies, and to provide initial $\mathrm{T}$ configurations for simulated heating experiments, we also simulate tangential director fields in thin and thick shells; see Appendix C.

Regarding the final step in observation 1 in the above list, we find multiple locally stable configurations but the experimentally detected $+1^{b},+1^{t} \mathrm{H}$ configuration has the lowest free energy for reasonable parameter values $1 / \xi_{R}=50$ $(R \approx 1 \mu \mathrm{m})$ and $\eta=4[t=-1.79, c=0.1, \rho=0.7]$. This conclusion holds generally for large $1 / \xi_{R}$ and large $\eta$. The
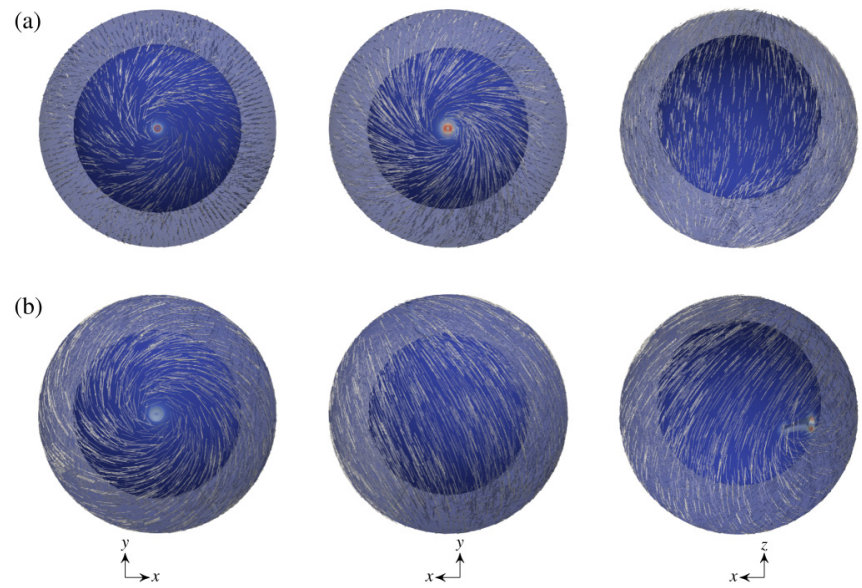

FIG. 5. Simulated trajectory of the $\mathrm{H} \rightarrow \mathrm{T}$ transition starting from a twisted $\mathrm{H}$ configuration $[t=-1.79, c=0.1, \rho=0.7$, and $\eta=4$ ]: (a) $n=1000$ and (b) 30000 , where $n$ denotes the number of iterations. Left to right: view from the thin part, the thick part, and side, respectively.

two +1 point defects on the inner, tangential interface move to antipodal points (as far away from each other as possible within geometrical constraints), to minimize the distortion energy of the director field.

Particularly interesting is that we find a twist throughout the shell for the $+1^{b},+1^{t} \mathrm{H}$ configuration [Fig. 4(a)], whereas a $2\left(+1 / 2^{b}\right),+1^{t} \mathrm{H}$ configuration [Fig. 4(b)] has the twist localized to the top half, hosting the +1 defect, in good agreement with experimental observations [visualized in Figs. 3(d)-3(f) and 3(g)-3(i), respectively]. Moreover, the simulations demonstrate that an $\mathrm{H}$ shell can exhibit $+1 / 2$ disclinations, albeit of a different geometry than in $\mathrm{T}$ shells. The disclinations running from the shell inside to the outside in the latter require both interfaces to be tangential; if the director were tilted out of the interface plane near the disclination, the $180^{\circ}$ rotation around $\mathrm{a}+1 / 2$ defect would invert the tilting direction, thus it would no longer be a symmetry operation. However, $+1 / 2$ disclinations can still exist as long as one interface is tangentially aligned, by curving the disclination line into the topology of a U, see Fig. 4(b), right. Visible $+1 / 2$ defects on $\mathrm{H}$ shells are then connected in pairs, comprising the start and end points of a U-turned disclination on the tangential boundary (the inner boundary in this case).

\section{Simulation of the $\mathrm{H} \rightarrow \mathrm{T}$ trajectory}

Regarding observation 4, we perform a gradient flow simulation at fixed temperature for a shell that has been set up with the equilibrium $+1^{b},+1^{t} \mathrm{H}$ configuration, switching to tangential anchoring at both interfaces at the start of the simulation. The results for $c=0.1, \rho=0.7$, and $1 / \xi_{R}=50$ $(R \approx 1 \mu \mathrm{m})$ with $\eta=4$ at $t=-1.79$ are shown in Fig. 5 . Initially, the +1 defect near the thinnest part of the shell appears stable, now as a +1 defect pair (one defect on the shell inside, one on the outside), see Fig. 5(a). At a later point, the +1 defect in the thick part breaks into two $+1 / 2$ defects, which move towards the thinnest part of the shell, see Fig. 5(b). Eventually, the remaining +1 defect pair becomes unstable, yielding a final $4\left(+1 / 2^{t}\right)$, or $\mathrm{T} 1$, state. 
The disagreement with the experimental results in Fig. 2 concerning the final stable configuration may have several explanations. While it could be related to the limited size of the simulated shell, a more important factor is probably that the experiment follows a trajectory through which the order parameter and, consequently, elastic constants, interfacial tension and boundary conditions vary continuously (see Discussion). As will become clear below, it is very likely that the alignment at one or both interfaces changes gradually during the experiment, thus passing through a region where a tilted alignment (neither tangential nor normal) is promoted, with possible exceptions in localized regions. In contrast, the simulation is done for constant temperature deep in the nematic phase, fixed elastic constants, and a suddenly imposed alignment change, from perfect $\mathrm{H}$ to perfect $\mathrm{T}$ conditions. Thus, the simulations find minima other than those observed in the experiments. Nevertheless, the numerical experiment captures the transient stability of at least one +1 defect pair and the tendency of all topological defects to migrate towards the thinnest part of the tangential shell. As will become clear in the Discussion, this simulation may be more relevant for the conditions that led to the appearance of the pristine T1 shells.

\section{B. Detailed analysis of tangential-hybrid-normal trajectories in E7 shells}

In the realignment trajectories described in Fig. 2, steps 2 and 3 always occur above the critical micelle temperature $\left(24^{\circ} \mathrm{C}\right)$ of a $1 \%$ aqueous F127 solution [64], hence we do not expect changes in the behavior of F127 to be at the origin of these steps. Moreover, the temperatures of 5CB shell realignment transitions discussed above are largely below the range in which realignment was detected in our preliminary study on $8 \mathrm{CB}$ shells; the relevant range in that case was $33^{\circ} \mathrm{C}-40^{\circ} \mathrm{C}$ [12]. These observations suggest that the realignment is not primarily due to thermally induced effects in the F127 solution but rather related to the variation of nematic order within the LC. To confirm this, we conduct experiments on shells prepared using E7 (supplementary movies M2-M4, Figs. 6, 8 , and 10 ), with a much higher $T_{\mathrm{NI}}$ than $5 \mathrm{CB}$.

An added benefit of the E7 shells is that the expanded nematic temperature range allows us to study each trajectory in more detail and, in contrast to the 5CB shell case, there are no overlapping transitions, not even when starting in a $\mathrm{T} 3 \mathrm{con}-$ figuration. As above, all shells are pristine at the beginning of the experiment, i.e., they have not been subject to temperature changes after the rapid cooling ending production. We only illustrate one shell at a time but similar experiments have been carried out on 20-50 E7 shells for each starting configuration, allowing us to be reasonably confident about the generality of our observations and conclusions. For selected panels we provide corresponding director field sketches. We refer to the right part of Fig. 2 for interpretation of the textures in general.

As shown in Appendix C, for a thin shell with $\xi_{R}=50$ $(R \approx 1 \mu \mathrm{m})$ as in the previous figures, we only observe the T3 $\left[4\left(+1 / 2^{t}\right)\right]$ configuration in numerical simulations. However, for thicker shells we can find three equilibrium $\mathrm{T}$ configurations. Therefore, in this section, our simulated shells (Fig. 7, 9 , and 11) have a relative thickness greater than that in experiments, in order to investigate the tangential-hybrid-

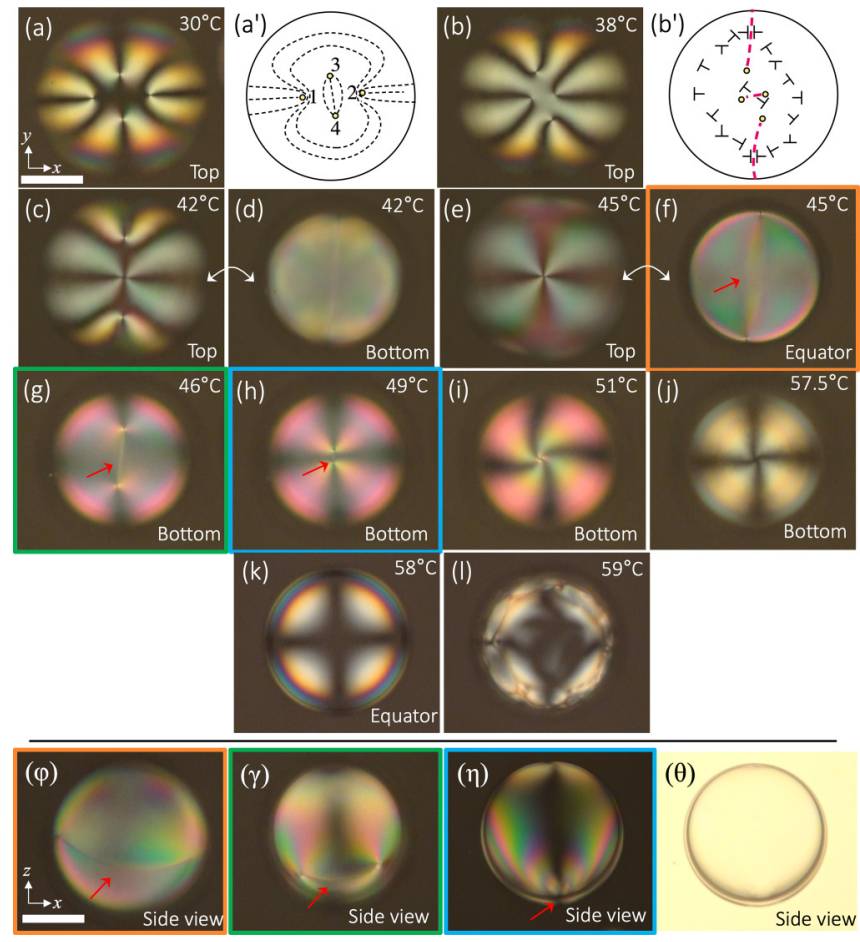

FIG. 6. Alignment transformation upon heating of an E7 shell starting in $\mathrm{T} 1$ configuration $\left[4\left(+1 / 2^{t}\right)\right]$, stabilized by F127 on both sides. The shell is between crossed polarizers (horizontal and vertical) except in panel $(\theta)$, obtained without analyzer. The focal plane is noted in each top view panel [(a)-(1), coordinate system in (a)] $(\phi),(\gamma),(\eta)$, and $(\theta)$ show side views (coordinate system in $\phi)$, obtained with the microscope tilted $90^{\circ} ;(\phi),(\gamma)$, and $(\eta)$ roughly correspond to (f), (g), and (h), respectively, as illustrated by the colored frames (for practical reasons, top and side views are from separate experiments). The ground state director field is drawn in $\left(\mathrm{a}^{\prime}\right)$. In $\left(b^{\prime}\right)$, the tilt direction is suggested with nails, nail head, and tail signifying upwards- and downwards-pointing director, respectively (or vice versa). The $\pi$ defect lines are drawn as dashed red lines in $\mathrm{b}^{\prime}$ and highlighted with red arrows in the micrographs. Scale bar: $50 \mu \mathrm{m}$.

normal trajectories starting from different $\mathrm{T}$ configurations also numerically.

\section{Realignment from T1 configuration $\left[4\left(+^{1 / 2^{t}}\right)\right]$}

The four initial defects are labeled 1-4 in Fig. 6(a'), corresponding to the micrograph in (a). To map out the director field with certainty, the shell is observed temporarily with a first-order $\lambda$ plate (see movie M2). As $\mathbf{n}$ on the shell outside starts tilting upon heating [Figs. 6(b)-6(d)], two faint lines appear. A short line [red dashed line in Fig. $6\left(b^{\prime}\right)$ ] connects defects 1 and 2 over the shell top, and a longer (red dashed line in $\left(b^{\prime}\right)$, highlighted by red arrows in micrographs) connects defects 3 and 4, running around the back of the shell. These lines can be interpreted as $\pi$ lines (see also Sec. IV A), separating regions with opposite directions of interface tilt, and thus of bend within the shell, during the $\mathrm{T}_{\text {heat }} \mathrm{H}$ transition. We indicate the tilt with nails in Fig. 6(b), where the nail head and tail can represent upwards- and downwards-pointing $\mathbf{n}$, respectively. However, as we cannot experimentally distinguish between 

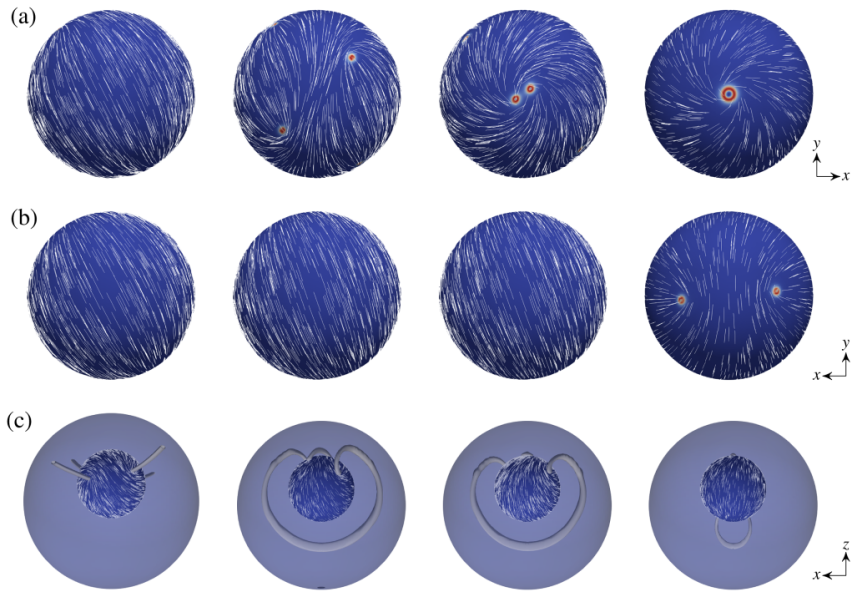

FIG. 7. Simulated trajectory of the $\mathrm{T} \rightarrow \mathrm{H}$ transition starting from $\mathrm{T} 1\left[4\left(+1 / 2^{t}\right)\right]$, with $t=-1.79, c=0.2, \rho=0.4$. and $\eta=4$. We plot $\mathbf{n}$ at the inner shell boundary [left to right: simulations steps $n=0,10000,15000$ and 20000]: (a) view from thin part, (b) view from thick part, and (c) side view. Defects are identified with the red regions or regions of high biaxiality.

the two possible tilt directions, the inverse interpretation is equally possible.

As heating continues, defects 1 and 2 move closer until we reach step 1 [(Figs. 3(a)-3(c)], where the defects settle at the shell top without merging [Fig. 6(e)]. They are so close to each
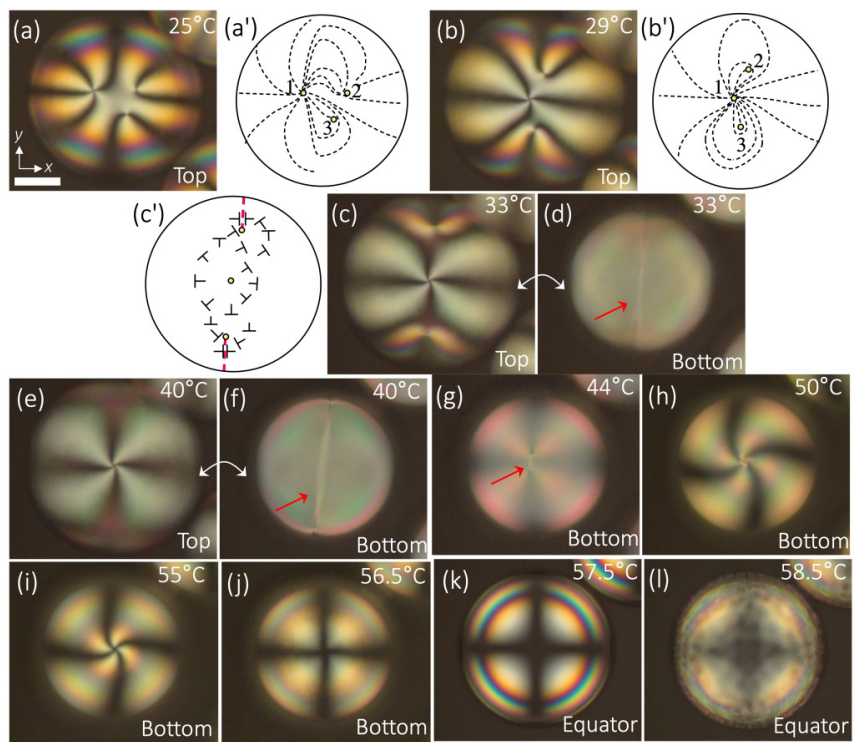

FIG. 8. Alignment transformation upon heating [(a)-(1)] of an E7 shell starting in the T2 configuration $\left[2\left(+^{1 / 2} 2^{t}\right),+1^{t}\right]$, stabilized by F127 on both sides. The shell is between crossed polarizers (horizontal and vertical). The focal plane is noted in each panel. We draw the ground state director field $\left(a^{\prime}\right)$ and the new one $\left(b^{\prime}\right)$ arising after a defect exchange that initiates the transformation. In $\left(c^{\prime}\right)$, the tilt direction is suggested with nails, nail head and tail signifying upwards- and downwards-pointing director, respectively (or vice versa). The $\pi$ defect line is highlighted with red arrows in the micrographs and its ends reaching the top shell half are drawn as dashed red lines in $\mathrm{c}^{\prime}$. Scale bar: $50 \mu \mathrm{m}$.
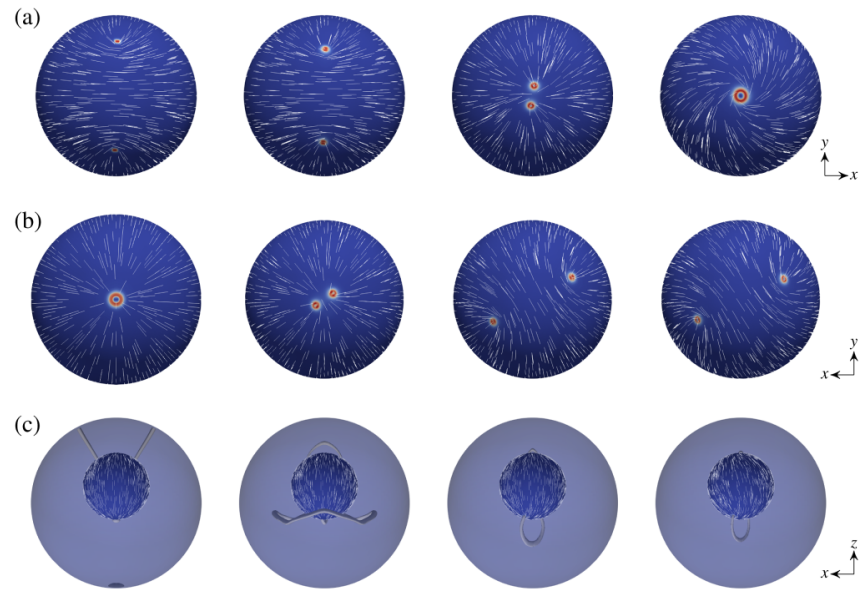

FIG. 9. Simulated trajectory of the $\mathrm{T} \rightarrow \mathrm{H}$ transition starting from T2 $\left[2\left(+1 / 2^{t}\right),+1^{t}\right]$, with $t=-1.79, c=0.2, \rho=0.4$, and $\eta=$ 4. We plot $\mathbf{n}$ at the inner shell boundary [left to right: simulation steps $n=0,5000,20000$, and 40000 ]: (a) view from thin part, (b) view from thick part, and (c) side view.

other that their separation can only be distinguished with perfect focus (movie M2). Defects 3 and 4 initially move further apart, accelerating after step 1 , reaching the equator in (f) and the bottom region in $(\mathrm{g})$. They merge [step 3, Figs. 3(g)-3(i)] between (h) and (i), immediately triggering a twisting of the surrounding bottom shell half director field recognized by a spiral pattern. Defects 1 and 2 must have merged into a +1 defect slightly earlier [step 2, Figs. 3(d)-3(f)], although the

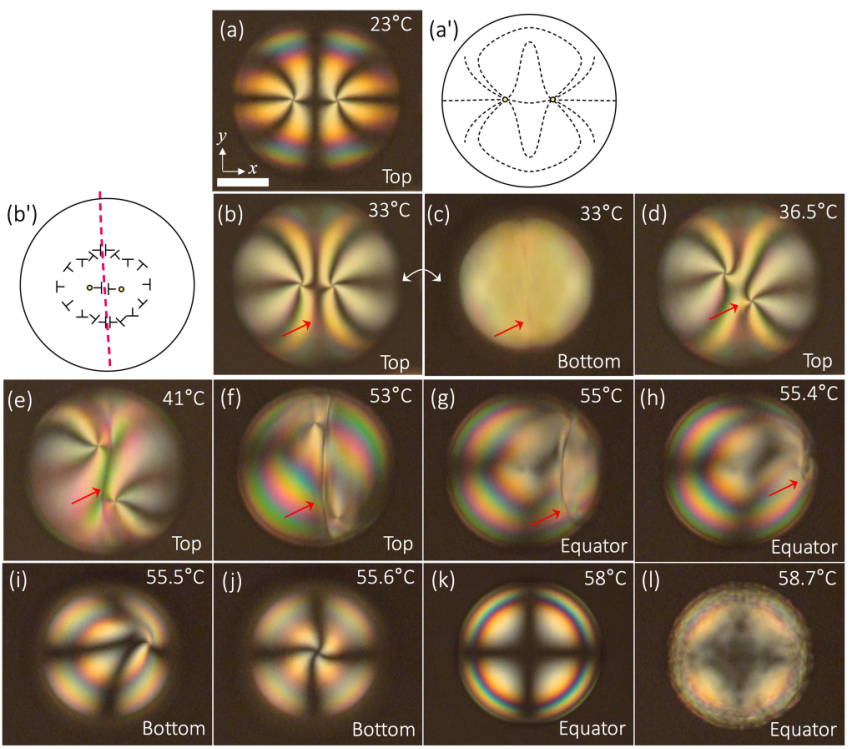

FIG. 10. Alignment transformation upon heating [(a)-(1)] of an E7 shell starting in T3 configuration $\left[2\left(+1^{t}\right)\right]$, stabilized by F127 on both sides. The shell is between crossed polarizers (horizontal and vertical). The focal plane is noted in each panel. The ground state director field is drawn in $\left(a^{\prime}\right)$. In $\left(b^{\prime}\right)$, the tilt direction is suggested with nails, nail head and tail signifying upwards- and downwardspointing director, respectively (or vice versa). The $\pi$ circle separating opposite tilt directions is drawn in red in $\mathrm{b}^{\prime}$ and highlighted with red arrows in the micrographs. Scale bar: $50 \mu \mathrm{m}$. 

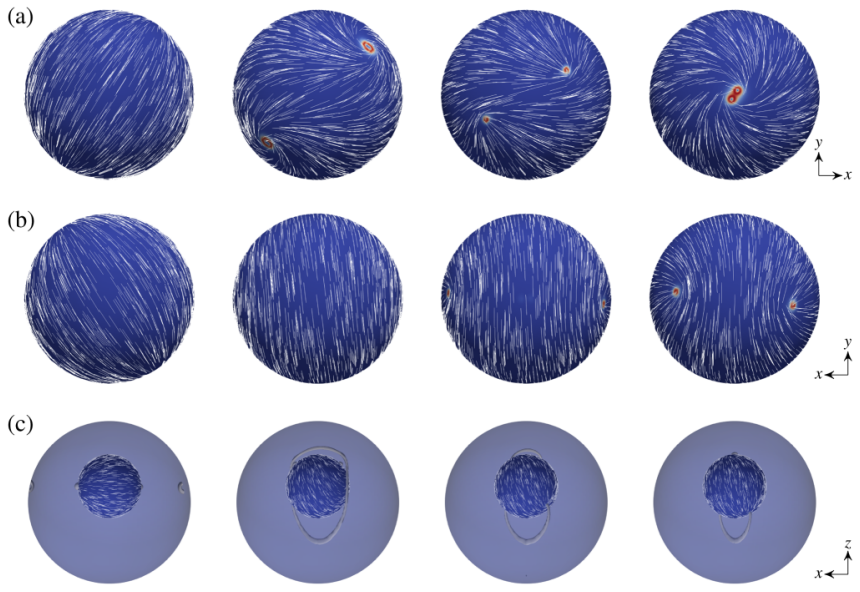

FIG. 11. Simulated trajectory of the $\mathrm{T} \rightarrow \mathrm{H}$ transition starting from a $+1^{l},+1^{r}$ (left, right) tangential configuration, with $t=$ $-1.79, c=0.2, \rho=0.4$, and $\eta=4$; we plot $\mathbf{n}$ on the inner shell boundary [simulation steps $n=0,30000,35000$ and 40000 from left to right]. (a) View from the thin part, (b) view from the thick part, and (c) side view.

event is not visible in Fig. 6/movie M2 because the focus stays at the shell bottom from (g) to (i). (It is seen, however, in two other E7 shells that start from the T1 configuration in movie M4.) In Fig. 6(i), the shell thus has a hybrid $+1^{b},+1^{t}$ configuration with twisted director field, the tangential side having the director field illustrated in Figs. 3(g)-3(i). The antipodal +1 defects disappear around $58^{\circ} \mathrm{C}$ [Figs. $6(\mathrm{j})$ and $6(\mathrm{k})]$ when the shell becomes fully normally aligned $\left(\mathrm{H}_{\text {heat }} \mathrm{N}\right)$. The shell clears at about $59^{\circ} \mathrm{C}$ [Fig. 6(1)].

Panels $(\phi-\theta)$ in Fig. 6 show representative micrographs of another shell initially in T1 configuration undergoing the same realignment trajectory upon heating, viewed from the side by tilting the microscope $90^{\circ}$. It is experimentally impossible to have both views at the same time, hence the shell in the side view in $(\phi)$, corresponding to the top view (f), has the defect line nearer the equator than the shell in (f). The defect locations in the side views in $(\gamma-$ $\eta$ ) correspond quite well to those in the top views in $(\mathrm{g})-$ (h), respectively. Panel $(\theta)$ shows the side view shell without analyzer, making it easy to see the asymmetry in shell thickness.

In Fig. 7, we employ the gradient flow algorithm to simulate the transient dynamics within a simplified framework, tracing a possible path towards a stable $\mathrm{H}$ state. The simulation is done for fixed temperature and material parameters and we do not consider tilted alignment at any interface, hence the outside boundary condition is suddenly switched to normal while the inside boundary remains tangential. The shell is asymmetric with thin top, outer radius of $1 \mu \mathrm{m}$ and LC elastic anisotropy parameter, $\eta=4$.

The transition starts with two $+1 / 2$ defects on the shell inside connecting via a short U-curved disclination line through the thin top of the shell, and the two others via a long curved disclination line extending around the back, see Fig. 7(c). Similar to the experiment, the first two approach each other, moving towards the top. However, upon meet- ing they merge immediately to yield $\mathrm{a}+1^{t}$ defect, hence in the simulation steps 1 and 2 coincide. The twisting of the simulated director field starts when the defects are very close, but before the merger, in contrast to experiment, see field plots 2 and 3 in Fig. 7(a). The remaining two $+1 / 2$ defects migrate to the thickest part and settle there, sufficiently separated so as not to induce a twisted director field in their shell half. In other words, we do not reproduce step 3 of the experiment nor do we reach the final $+1^{b},+1^{t}$ hybrid configuration with a twisted director field throughout the shell.

We identify three main reasons for the differences between experiments and simulations regarding the trajectory as well as the final state. Firstly, the simulations are not carried out as a function of temperature, instead triggering the realignment by switching the outer boundary from hard tangential to hard normal, while the inside remains hard tangential throughout. Secondly, compared to the experimental shells, the simulated shells are small and relatively thick, since computational resolution renders it difficult to extract structural information for thinner shells. Finally, these complex systems have multiple stable configurations [65] and the gradient flow algorithm may converge to a locally stable configuration. Indeed, our direct simulations of hybrid director fields in Fig. 4 produce a $+1^{t}, 2\left(+1 / 2^{b}\right)$ configuration with twisted director field through the shell top and $\mathrm{a}+1^{b},+1^{t}$ state with twist throughout the shell, the latter corresponding to the global free energy minimum. As the $+1^{t}, 2\left(+\frac{1}{2^{b}}\right)$ state is a local energy minimum, our dynamical algorithm converges to it and stays there, unable to overcome the energy barrier needed to reach the $+1^{b},+1^{t}$ state.

\section{Realignment from T2 configuration $\left[2\left(+1 / 2^{t}\right),+1^{t}\right]$}

In this realignment sequence, a preliminary step is a brief merger and immediate re-splitting of the +1 and one of the $+1 / 2$ defects $\left[1\right.$ and 2 in Fig. 8( $\left(a^{\prime}\right)$ ], rearranging the surrounding director field as sketched in Fig. 8( $\left.\mathrm{b}^{\prime}\right)(\lambda$-plate appearance in movie M3). The resulting +1 defect moves towards the top, reaching it in panel $\left(\mathrm{c} / \mathrm{c}^{\prime}\right)$ with the director field twist in the top half of the shell immediately seen [step 2, Figs. 3(d)-3(f); no step 1 in this sequence). The remaining trajectory, Figs. 8(d)8(1), is very similar to that of Sec. III B 1.

The corresponding gradient flow dynamics simulation (asymmetric shell, dimensions and simulation conditions as above) produces quite a different trajectory (Fig. 9) compared to the experiment, in particular for the original +1 point defect on the inner surface. It first moves to the thick shell side where it splits into two $+1 / 2$ defects connected by a wide U-turned disclination loop, see Fig. 9(b). The two original $+1 / 2$ defects (connected by a U-turned disclination loop after the outer interface alignment is switched to normal) move to the thinnest point, where they merge into $\mathrm{a}+1$ defect with a twisted director field around it, see row (a). Their behavior is thus closer to what is seen in experiment, albeit on the opposite shell side.

The final $\mathrm{H}$ state is again $+1^{t}, 2\left(+\frac{1}{2^{b}}\right)$, since the two $+1 / 2$ defects on the thick side of the shell do not merge. As above, the discrepancies compared to the experimental observations 
are most likely due to the simplifications of the simulation conditions and to the gradient flow simulation getting stuck in a local energy minimum.

\section{Realignment from T3 configuration $\left[2\left(+1^{t}\right)\right]$}

Compared to the cases where $+1 / 2$ disclinations are initially present, the first signs of response of an E7 shell initially in T3 configuration [Figs. 10(a) and $10\left(\mathrm{a}^{\prime}\right)$ ] are seen at a later stage, just like for the corresponding 5CB shells (Fig. 2). By about $33^{\circ} \mathrm{C}$ the colors have changed and a $\pi$ defect circle can be recognized, see Figs. 10(b), 10(b'), 10(c), and movie M4. The circle arises half-way between the two +1 defects, aligned normal to the line separating them. At $35^{\circ} \mathrm{C}$ [between panels (c) and (d) in Fig. 10], the two +1 defects start moving in opposite directions along the $\pi$ circle. As for the case of 5CB shells, we define this event as step 1 in the T3-initiated trajectory. We remind that the three steps starting from T3 are not directly comparable with those starting from $\mathrm{T} 1$ or T2. The defects move further apart [Fig. $10(\mathrm{e})]$ and at $53^{\circ} \mathrm{C}$ [Fig. 10(f)] the circle starts moving rightwards, pushing one of the +1 defects ahead of it. The other +1 defect detaches (step 2) and retracts to the shell top in panel (g).

At $55.4^{\circ} \mathrm{C}$ [Fig. $10(\mathrm{~h})$ ], the $\pi$ circle has almost closed in upon itself, the trapped +1 defect pushed to the far right. Soon after, the circle disappears (step 3), allowing the previously trapped defect to move down to the bottom of the shell [Figs. 10(i) and 10(j)]. In contrast to the trajectory with 5CB shells, we here obtain the usual $+1^{b},+1^{t} \mathrm{H}$ configuration at $55.6^{\circ} \mathrm{C}$ [Fig. 10(j)], with a twisted director field throughout the shell. Heating yet a bit more, the shell acquires an $\mathrm{N}$ configuration at $58^{\circ} \mathrm{C}$ [Fig. $\left.10(\mathrm{k})\right]$. The shell clears at $58.7^{\circ} \mathrm{C}$ [Fig. 10(1)]. For all shells starting in T3, whether E7 or 5CB, our $\mathrm{T}_{\text {heat }} \mathrm{H}$ trajectory is identical. It corresponds to the second scenario discussed by Lopez-Leon in Ref. [7].

We are not able to reproduce the $2\left(+1^{t}\right)$ tangential configuration in simulations (see Appendix C), hence we compare the experimental observations with simulations from a slightly different initial T3' configuration, with two +1 defect pairs on the sides (Fig. 11). The shell retains the same asymmetry and dimensions as before, with $\eta=4$, and the simulation is done in the same way with a sudden switch from tangential to normal anchoring on the outside interface.

In the initial stages of the dynamical evolution, the two +1 defects approach and the surrounding director field twists simultaneously, yielding a situation analogous to the experiment in Figs. 10(d) and 10(e). However, in stark contrast to the experimental trajectory, we then observe a splitting of the two +1 defects into four $+1 / 2$ defects, connected pairwise by U-bent disclinations through the shell. Two $+1 / 2$ defects then merge into $\mathrm{a}+1^{t}$ defect with surrounding twisted director field at the thinnest part of the asymmetric hybrid shell. The remaining two $+1 / 2$ defects migrate to the thickest part of the shell, again without merging. As above, we attribute the discrepancies between simulation and experiment to the differences in shell size and the nonvarying LC parameters.

\section{Detailed investigation of hybrid shells}

The experiments in Secs. III A and III B show that the temperature $T_{c}$ when the gradual change from tangential to

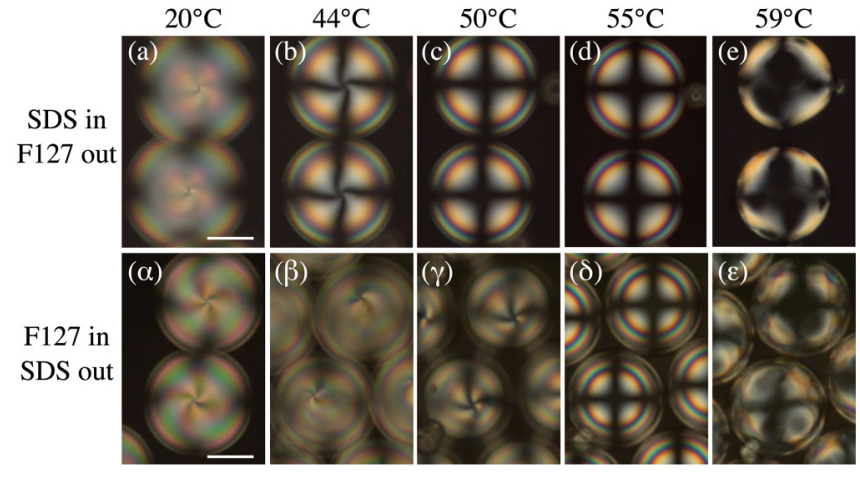

FIG. 12. Heating E7 shells stabilized by the normal-aligning surfactant SDS on the inside and F127 on the outside [(a)-(e)] or the other way around $[(\alpha)-(\epsilon)]$, from $20^{\circ} \mathrm{C}$ to the clearing point. All shells start out in H configuration, the F127-stabilized side tangential. With F127 on the outside, the shells switch to $\mathrm{N}$ configuration above $44^{\circ} \mathrm{C}\left[(\mathrm{b})\right.$ and (c)], whereas this transition is seen only at about $55^{\circ} \mathrm{C}$ when $\mathrm{F} 127$ is on the inside $[(\gamma)$ and $(\delta)]$. The tangential-aligning ability of F127 is thus weaker on the outside than on the inside. Scale bars: $50 \mu \mathrm{m}$.

normal anchoring has completed is different on the shell inand outside, as otherwise we would not get the $\mathrm{H}$ configuration at intermediate temperatures. But these experiments do not reveal which side has lower $T_{c}$. In order to elucidate this issue, we conduct a series of reference experiments where we stabilize shells only from one side with F127, the opposite side stabilized by a $1 \%$ SDS solution. It is well established that SDS at this concentration induces strong normal anchoring, hence the SDS-stabilized side is always normal. As the F127stabilized side is predominantly tangential at room temperature, all shells in the reference experiments start out in $\mathrm{H}$ configuration. On heating, the F127-stabilized side gradually changes anchoring, the shell switching to $\mathrm{N}$ configuration when $T>T_{c}$. If $T_{c}$ is lower on the inside, this happens at a lower temperature than when we reverse the geometry. If $T_{c}$ is lower on the outside, it will be the other way around.

The results are shown in Fig. 12 and in movies M5 and M6. As predicted, all shells start out in $\mathrm{H}$ configuration at $20^{\circ} \mathrm{C}$, see panels (a) and $(\alpha)$. The texture changes gradually upon heating and at $44{ }^{\circ} \mathrm{C}$ the shells with $\mathrm{F} 127$ on the outside and SDS on the inside acquires an N configuration, see Fig. 12(b). The shells with the inverted stabilizer geometry still have a complex $\mathrm{H}$ texture at this temperature [Fig. 12( $\beta)$ ], turning into $\mathrm{N}$ only at $55^{\circ} \mathrm{C}$ [Fig. $12(\delta)$ ], 10 degrees higher. The comparison clearly shows that F127 retains its tangentialaligning influence to higher temperatures on the inside than on the outside, and we can thus conclude that all $\mathrm{H}$ shells described in Secs. III A and IIIB have the outside close to normal anchoring; the tilt gradually increases, reaching normal alignment at a temperature for which the inside still has tilted (or tangential) alignment.

To get further details with 3D resolution, we investigate four E7 shells in $+1^{b},+1^{t} \mathrm{H}$ configuration, stabilized by F127 on both sides, by fluorescent confocal polarizing microscopy (FCPM). As seen in the cross-sectional image sequence of Figs. 13(a)-13(c) and supplementary movie M7, the fluorescence emission reveals a twisted director field from bottom to 

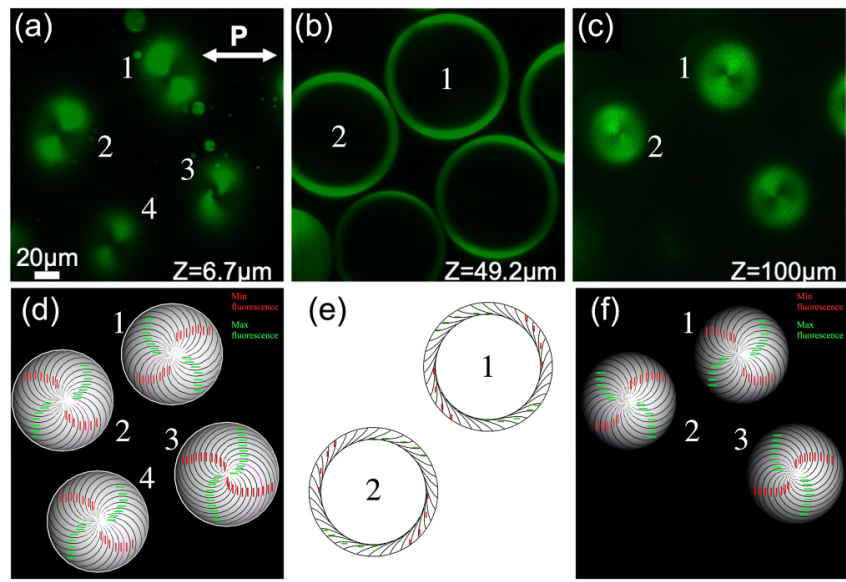

(e)
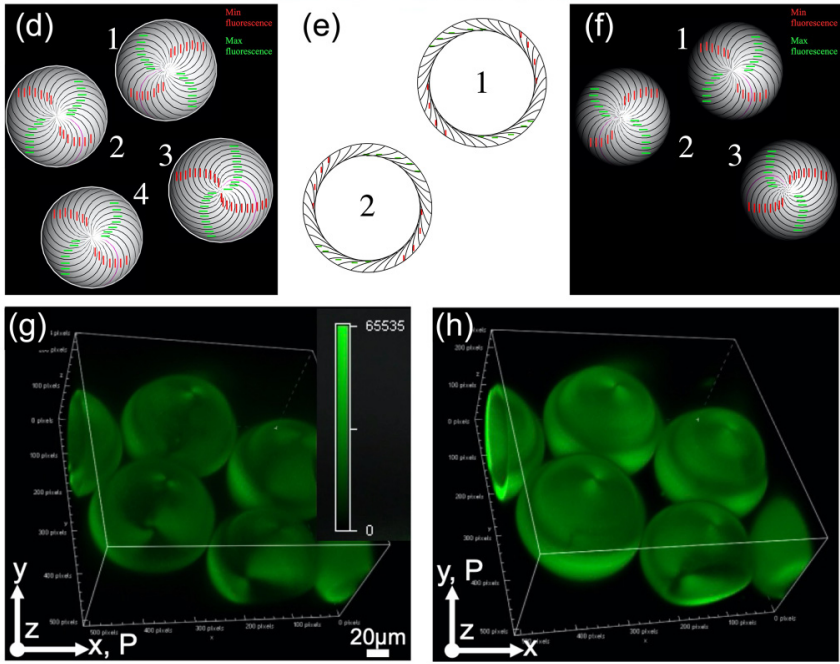

FIG. 13. FCPM images of E7 shells in H configuration stabilized by $1 \% \mathrm{~F} 127$ on in- and outside, taken at $54{ }^{\circ} \mathrm{C}$. Fluorescence images and corresponding director field sketches (for connecting top to bottom, one field line has been colored pink) are for cross sections near the shell bottom [(a) and (d), $z=6.7 \mu \mathrm{m}]$, middle plane [(b) and (e), $z=49.2 \mu \mathrm{m}$ ], and top [(c) and (f), $z=100 \mu \mathrm{m}$ ]. For clarity, we draw only shells 1 and 2 in (e). Locations with $\mathbf{n}$ parallel to the exciting laser polarization $\mathbf{P}$ (maximum fluorescence from the BTBP dye) are highlighted with green lines, whereas locations with $\mathbf{n} \perp \mathbf{P}$ (minimum fluorescence) are indicated with red lines. All shells are qualitatively similar to Figs. 3(g)-3(i), although shells 2-4 have the opposite handedness and the degree of twist varies, with shell 4 having stronger twist than 1-3. [(g) and (h)] 3D volume-views of the shells, for $\mathbf{P}$ along $x$ (g) and $y(\mathrm{~h})$, respectively. The upper half of each shell appears with artificially reduced thickness due to refraction at the water-LC interface at the shell bottom.

top. If there were no twist, the fluorescence maxima would be along $\mathbf{P}$ and the minima $\perp \mathbf{P}$. We note that the fluorescence patterns at the bottom [Figs. 13(a) and 13(d)] and the top [Figs. 13(c) and 13(f)] are each others' mirror images. This is because the fluorescence intensity reflects the component of the dye molecule's transition moment along the exciting light polarization. This is given by the projection into the horizontal plane of the orientation of the dye molecule, and thus of the director field. As illustrated by comparing panels (d) and (f) in Fig. 13, this projection at the top is the mirror image of that at the bottom. This is also seen in Fig. 3(g), showing the microscopy perspective of an $\mathrm{H}$ configuration shell, with direct comparisons with other perspectives. This means that the apparent projected spiral director field around each +1 defect appears with opposite handedness at the top and bottom for the same shell.

At the shell mid plane [Figs. 13(b) and 13(e)], the projection of $\mathbf{n}$ into the horizontal plane exhibits a bend from the
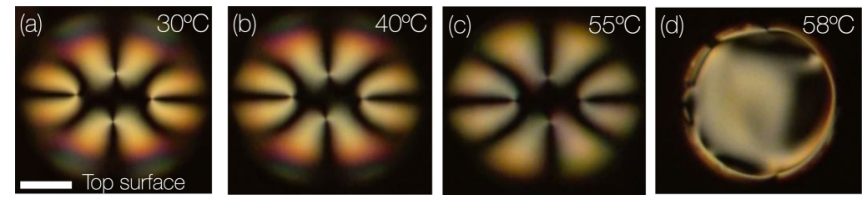

FIG. 14. E7 shells stabilized by aqueous PVA solutions on both sides, heated from room temperature to $T_{\mathrm{NI}}$. The shells remain in a T3 $\left[4\left(+\frac{1}{2^{t}}\right)\right]$ configuration (a)-(c) until clearing (d). Scale bar: $50 \mu \mathrm{m}$.

predominantly tangential inside to the predominantly normal outside. Both interfaces are likely to have tilted alignments; if the outside were perfectly normal, the bend would take place over a rather small distance and we should see strong fluorescence at the top and bottom (of the image, not of the shell) along the shell insides, and on the left and right along the shell outsides, but this is not what we see. The rotated maxima and minima indicate tilted alignments with respect to the interface normals. In Figs. 13(g) and 13(h), the full 3D FCPM images are shown for two perpendicular orientations of the exciting light polarizations. In $(\mathrm{g})$, the polarization is identical to the cases in (a)-(c), whereas (h) has the perpendicular polarization.

\section{Comparison with PVA as stabilizer}

To confirm that the temperature-induced alignment change is indeed characteristic of shells stabilized by the block copolymer F127, we also produce E7 shells stabilized by $1 \%$ aqueous solutions of $85 \%$ hydrolyzed PVA on both sides, heating the shells at $5 \mathrm{~K} /$ minute from room temperature to $T_{\mathrm{NI}}$, see movie M8 and Fig. 14. The T1 configuration texture remains intact constantly throughout the heating process, until clearing starts at $T \approx 58^{\circ} \mathrm{C}$, see Fig. 14 . The only change that can be distinguished prior to clearing is a slight decrease in birefringence.

\section{DISCUSSION}

We summarize all experimental alignment transition temperatures for 5CB and E7 in Table I. In order to compare the two LCs and to relate the transitions to the degree of orientational order rather than to a fixed temperature scale, we use an experimental reduced temperature $T_{r}=\left(T-T_{\mathrm{NI}}\right) / T_{\mathrm{NI}}$, with $T$ and $T_{\mathrm{NI}}$ in Kelvin. All transitions, regardless of starting configuration, take place at somewhat lower $T_{r}$ in E7 than in

TABLE I. Experimental reduced alignment transition temperatures $T_{r}=\left(T-T_{\mathrm{NI}}\right) / T_{\mathrm{NI}}$ for $5 \mathrm{CB}$ and E7 shells. The three steps of the $\mathrm{T} \rightarrow \mathrm{H}$ transition are defined above. The final transition is from hybrid to normal, $\mathrm{H} \rightarrow \mathrm{N}$.

\begin{tabular}{lccccc}
\hline \hline LC & Start. config. & $T_{r}^{\text {step 1 }}$ & $T_{r}^{\text {step } 2}$ & $T_{r}^{\text {step 3 }}$ & $T_{r}^{\mathrm{H} \rightarrow \mathrm{N}}$ \\
\hline 5CB & T1 & -0.040 & -0.013 & -0.006 & -0.001 \\
E7 & T1 & $-\mathbf{0 . 0 8}$ & $-\mathbf{0 . 0 5}$ & $-\mathbf{0 . 0 3}$ & $-\mathbf{0 . 0 0 5}$ \\
5 CB & T2 & - & -0.034 & -0.0058 & -0.001 \\
E7 & T2 & - & $-\mathbf{0 . 0 8 1}$ & $-\mathbf{0 . 0 3 7}$ & $-\mathbf{0 . 0 0 5}$ \\
5 CB & T3 & -0.034 & -0.002 & - & -0.001 \\
E7 & T3 & $-\mathbf{0 . 0 7 2}$ & $-\mathbf{0 . 0 2 7}$ & $-\mathbf{0 . 0 1 3}$ & $-\mathbf{0 . 0 0 6}$ \\
\hline \hline
\end{tabular}


5CB and all realignment transitions take place at higher $T_{r}$ when starting from T3 $\left[2\left(+1^{t}\right)\right]$ than from T1 $\left[4\left(+1 / 2^{t}\right)\right]$ or $\mathrm{T} 2\left[2\left(+1 / 2^{t}\right),+1^{t}\right]$. The latter discrepancy is not surprising, considering that the realignment trajectory starting from T3 is so different from those starting from $\mathrm{T} 1$ or $\mathrm{T} 2$. The three transition steps are thus not directly comparable between T1/T2 and T3. Nevertheless, for a given LC material, i.e., either 5CB or E7, Step 1 of the $\mathrm{T}_{\text {heat }} \rightarrow \mathrm{H}$ transition occurs at similar $T_{r}$ for the T1 and the T3 starting configurations. Note that the nonreduced temperatures are much more different between the two LCs (about $24^{\circ} \mathrm{C}$ for $5 \mathrm{CB}$ and about $35^{\circ} \mathrm{C}$ for E7).

For both LCs and all starting configurations, there is significant variation in $T_{r}$ of step 2, after which a half-shell director field twist arises. As for step 3, on the other hand, after which the entire shell adopts a twisted $+1^{b},+1^{t} \mathrm{H}$ configuration, we find similar $T_{r}$ for starting configurations $\mathrm{T} 1$ and $\mathrm{T} 2$, for a given LC material. The transition to normal $\left(\mathrm{H}_{\text {heat }} \mathrm{N}\right)$ takes place very near the clearing point $\left(T_{r}=0\right)$ in all shells, at a $T_{r}$ that is, within experimental error, independent of the starting configuration. Measured in normal temperature scale, the $\mathrm{H}_{\text {heat }} \mathrm{N}$ transition takes place at very different values, about $35.2^{\circ} \mathrm{C}$ for $5 \mathrm{CB}$ and about $57.3^{\circ} \mathrm{C}$ for $\mathrm{E} 7$.

\section{A. Why is the alignment tangential far below $T_{\mathrm{NI}}$ but normal near $T_{\mathrm{NI}}$ ?}

While it is experimentally challenging to distinguish tangential from slightly tilted anchoring, our assumption is that both shell interfaces are initially tangentially aligned at room temperature, thus giving us true $\mathrm{T}$ shells to start with. This assumption is based on the fact that we see no qualitative differences between the room temperature shell textures of shells stabilized by F127 and by PVA [compare Figs. 6(a) and 14(a)], respectively, the latter of which is well known to give strong tangential anchoring. Most significantly, the prevalence of the $\mathrm{T} 1$ and $\mathrm{T} 2$ configurations at room temperature, exhibiting two or four $+1 / 2$ disclinations, respectively, shows that the alignment on both in- and outside is tangential, at least in the vicinity of the disclinations.

We see textural changes upon heating and assume, based on the experiments in Fig. 12, that the alignment on the shell outside tilts away from the tangential conditions before the shell inside, and the tilt magnitude increases with increasing temperature. If tangential anchoring is lost everywhere on the shell outside, it can no longer support $+1 / 2$ disclinations. Hence the $\pi$ lines discussed in Figs. 6 and 8, connecting pairs of $+1 / 2$ defects, must then coincide with the U-turned disclinations within the shell(as observed in simulations). An alternative may be that the outside remains tangentially aligned locally around the $+1 / 2$ defects and the tilt appears only in the defect-free regions. The shell inside remains tangentially aligned for a longer length of time, but eventually allows for tilted alignment and then normal alignment, phase-shifted compared to the shell outside.

The experiments in this paper, together with those reported previously for $8 \mathrm{CB}$ in [12], show that this gradual realignment is not a function solely of absolute temperature, but it depends strongly on the LC. While $T_{r}$ for a certain transition listed in Table I is not identical for 5CB and E7 shells, it is always of the same order of magnitude. It is similar enough for the transition to be driven primarily by a change in the LC order. A secondary, much weaker, influence may be linked to temperature dependent behavior of the surrounding F127 solution, in particular regarding the degree of hydration of its different blocks (see below), explaining why a certain $T_{r}$ is always lower for $\mathrm{E} 7$ (high $T_{\mathrm{NI}}$ ) than for $5 \mathrm{CB}$ (low $T_{\mathrm{NI}}$ ).

F127 is an amphiphilic block copolymer. In contrast to low molar mass amphiphiles, which give normal alignment due to their radial alignent at the shell-water interface unless the concentration is very low [41], the impact of a block copolymer is more ambiguous. F127 has two hydrophilic PEO blocks, which are well hydrated near room temperature [64,66,67], thus bringing water in contact with the shell. This promotes tangential alignment $[43,68]$. In contrast, the hydrophobic PPO block at the center of the molecule is not hydrated. Its aliphatic nature promotes contact with the alkyl chain of LC molecules, favoring normal alignment. Because all three F127 blocks will be in contact with the LC shell, the overall influence on $\mathbf{n}$ at the LC boundaries is weak; two blocks favor tangential alignment, but they also prefer the water phase to the LC, and one block, preferring the LC, favors normal alignment. F127 is thus immensely interesting as it provides good shell stability through its adsorption at the LC-water interface, yet it gives little preference between tangential and normal alignment.

The anchoring of $\mathbf{n}$ at the shell boundary is dictated by the interfacial free energy density $F_{S}$ [see Eqs. (9) and (12)], i.e., $F_{S}=W\left(\mathbf{Q}-\mathbf{Q}^{\perp}\right)^{2}$, where $\mathbf{Q}^{\perp}$ is the tangential projection on the plane of the boundary and $W$ is a measure of the anchoring strength. In particular, if $W>0$, then tangential alignment is favoured so that $\mathbf{Q}=\mathbf{Q}^{\perp}$ whereas $W<0$ favours normal alignment. We conjecture that, for F127, $W>0$ but with low magnitude, proportional to $S_{b}$, where $S_{b}$ is a bulk scalar order parameter that can be computed from the minimizers of the bulk energy in Eq. (12). The slight emphasis for tangential alignment can be understood by considering the two large PEO blocks compared to the single smaller PPO block. Far below $T_{\mathrm{NI}}$, where $S_{b} \approx 0.7, W$ is large enough to impose tangential alignment despite the elastic energy penalty imposed by the resulting topological defects. However, as $T$ approaches $T_{\mathrm{NI}}$ on heating, $S_{b}$ decreases significantly and therefore $W$ decreases. The energetic penalty of not adopting tangential alignment on the outer shell surface decreases compared to the elastic energy cost of a tangential configuration with defects. This would qualitatively explain why the alignment gradually tilts over from tangential at room temperature to normal near $T_{\mathrm{NI}}$.

To test if this conjecture is plausible, we numerically simulate the director field of a shell with strong tangential anchoring on the inside but very weak tangential anchoring on the outside $\left(W=10^{-10} \mathrm{~J} \mathrm{~m}^{-2}\right)$, see Fig. 15 . Indeed, this results in an $\mathrm{H}$ configuration essentially identical to that in Fig. 4(a), obtained with strict normal anchoring on the outside.

Why does the realignment process start and finish earlier on the outside than on the inside? We propose that the negative curvature on the shell inside forces the LC to be more in contact with the hydrophilic blocks on the inside than on the outside. While for small molecules the curvature of a $100 \mu \mathrm{m}$ diameter shell may be considered negligible, this 

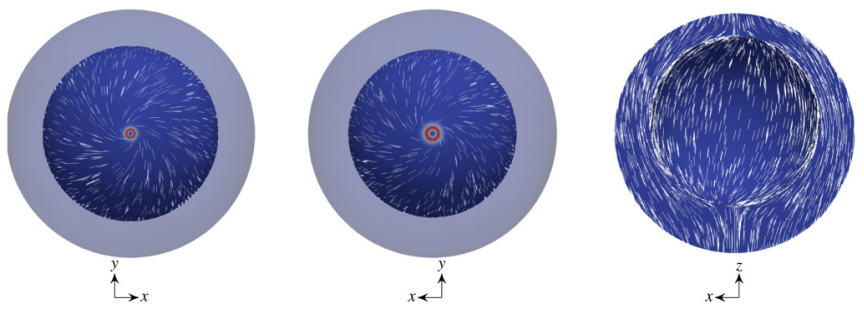

FIG. 15. Hybrid shell obtained with weak tangential anchoring on the outside $\left(W=10^{-10} \mathrm{~J} \mathrm{~m}^{-2}\right)$ and strong tangential anchoring on the inside $\left(W=10^{-2} \mathrm{~J} \mathrm{~m}^{-2}\right.$ ), viewed from the thinnest part (top), thickest part (bottom), and side, respectively, from left to right. A twisted $+1^{b},+1^{t}$ director field arises, similar to Fig. 4(a), with normal outer anchoring.

is not necessarily the case for molecules on the scale of a block copolymer. On the shell outside, with positive curvature, the LC around a point of contact with a PPO block curves away from the surrounding PEO blocks. They can thus avoid LC contact reasonably well, easily fluctuating outwards into the continuous aqueous phase, improving their hydration and increasing the entropy. This increases the packing density of PPO blocks on the LC shell outside, weakening the tangentialaligning influence of PEO blocks.

On the inside, in contrast, the LC around a point of contact with a PPO block curves towards the surrounding PEO blocks. The hydrated PEO blocks are thus more likely to be in contact with the LC on the inside than on the outside, strengthening the tangential-aligning influence there. Our conjecture needs to be corroborated and we hope our results can inspire thrusts with molecular dynamics simulations.

An alternative explanation has been proposed by Durey and Lopez-Leon in a reprint that we were recently made aware of Ref. [69], and previously also in a study by Lopez-Leon and coworkers focusing on cholesteric shells [23]. They report a similar change in alignment as described above, for PVAstabilized shells very near $T_{\mathrm{NI}}$, on heating at $0.01 \mathrm{~K} / \mathrm{min}$. We do not see this in our reference experiments with PVA (Fig. 14 and movie M8), probably due to faster heating. In Ref. [69], the authors propose that a transition to normal anchoring is driven by a thin surface layer of LC material that turns isotropic. We find this explanation unlikely, however, as the isotropic-nematic interface in cyanobiphenyl-based mixtures, with co-existing isotropic and nematic phases, has been shown to induce tangential alignment [70].

We do not know the degree of hydrolysis of the PVA used in Ref. [69], but as it is typical to use $85 \%$ hydrolyzed PVA for stabilizing LC shells (fully hydrolyzed PVA tends to give poor interface stability), we assume that this may have been the case. The remaining $15 \%$ are then polyvinylacetate which is less hydrated than polyvinylalcohol. While it is not a block copolymer, there is a variation in hydration between sections also in this stabilizer, and this could, qualitatively, give rise to the same features as discussed above. However, compared to F127 the tangential-aligning influence of PVA, even if not fully hydrolyzed, is much stronger, explaining why Durey and Lopez-Leon observed realignment only over tenths of degrees from the clearing point, whereas we see it over tens of degrees with F127-stabilized shells.

\section{B. Why do we see twisted director fields around +1 point defects in hybrid configurations}

A common feature of the experiments and simulations are twisted director fields around the +1 point defects on the tangential inner surface, for hybrid configurations. We believe that the twist is a consequence of the elastic anisotropy, i.e., when the splay elastic constant is larger in magnitude than the twist elastic constant. The elastic anisotropy is captured by the parameter $\eta=2\left(\frac{K_{1}}{K_{2}}-1\right)$. Our simulations show pure splay director fields around the +1 point defects for $\eta=0$ and the twist naturally appears on increasing $\eta$. The modeling at this stage does not capture the temperature dependence or even the material-dependence of the elastic constants. We also refer the readers to relevant calculations and observations by Williams [55] and by Lavrentovich et al. [71]. For example, in Ref. [55], the author works with full tangential droplets and shows that twisted (nonsplay) profiles around the +1 -defects are preferred for approximately $\eta>2.5$. We choose $\eta=4$, so that we are safely in a regime where pure splay profiles are energetically expensive compared to nonsplay profiles, thus favoring twisted defect profiles. This will be pursued more systematically in future work.

The first +1 point defect to form in a hybrid configuration always localizes at the thinnest point of the shell, in both experiments and simulations. For topological reasons, we must have a total topological charge of +2 on the tangential inner surface. In terms of the modeling, we find two competing hybrid configurations, $+1^{t},+1^{b}$ and $+1^{t}, 2\left(+1 / 2^{b}\right)$, both of which are local energy minimizers. In the experiments, we only observe the $+1^{t},+1^{b}$ hybrid configuration. The results are sensitive to a number of geometrical, material and environmental factors and since the modeling is largely phenomenological, we do not expect perfect agreement with experiments at this stage. We speculate that $+1^{t}, 2\left(+1 / 2^{b}\right)$ will lose its stability when the radius of the inner sphere becomes large enough, the thickness of the thick part becomes smaller, or $\eta$ is larger. This will be investigated in future work.

\section{What determines the tangential director field?}

The simulations reveal (Appendix C) that the energyminimizing tangential configuration is $\mathrm{T} 1\left[4\left(+1 / 2^{t}\right)\right]$, yet not all of the freshly prepared shells are in this configuration, some $15 \%$ showing the $\mathrm{T} 2\left[2\left(+1 / 2^{t}\right),+1^{t}\right]$ configuration and about $10 \%$ the $\mathrm{T} 3\left[2\left(+1^{t}\right)\right]$ configuration. In contrast, when the shells are cooled relatively slowly, going through the full sequence of realignment, all shells adopt the T3 configuration. Additional experiments (not shown) demonstrate that this is independent of shell thickness, and that it is stable in time (no change within 2 days confirmed).

We propose that each of the $\mathrm{T}$ configurations corresponds to a local energy minimum, with energy barriers for change from one to the other that are significantly greater than thermal energy at room temperature. When the shells are cooled slowly, the route via the $+1^{b},+1^{t}$ hybrid state templates a $\mathrm{T}$ state with two +1 defects, which move up to the thinnest point of the shell to form a $2\left(+1^{t}\right)$ T3 configuration once the tangential state is fully developed. Moreover, as long as a tilted alignment prevails across an interface, no $+1 / 2$ disclinations can form at that interface, hence only the T3 configuration is 
compatible with a shell that may appear like a true $\mathrm{T}$ shell but actually has a very weakly tilted interface alignment, away from tangential across either or both interfaces.

However, if the shells are quench cooled very rapidly, as is the case at the end of the shell production, the isotropicnematic phase transition takes place during strongly nonequilibrium conditions, and a random distribution of all possible $\mathrm{T}$ configurations is seen. The isotropic state may be temporarily supercooled to near room temperature, ensuring that the stable interface alignment is truly tangential once the nematic order develops, allowing $+1 / 2$ disclinations to form. This would explain why we see all $\mathrm{T}$ configurations in pristine shells. In fact, the simulations at constant LC parameters may in some respects be closer to these experimental conditions. Again the energy barriers for changing from one configuration to the other prevents a shell with a certain configuration to switch to another configuration, even if the other configuration has lower free energy.

\section{CONCLUSIONS AND OUTLOOK}

We have undertaken a comprehensive study of nematic shells stabilized by the amphiphilic block copolymer F127, using two different LC materials: 5CB and E7. Our most notable findings focus on the dynamic tuning of the boundary conditions for the shells, using temperature as the control variable. We experimentally record highly informative director field reconfigurations from tangential to hybrid and then to normal, before a transition to the isotropic phase, through carefully controlled heating experiments. Subsequent cooling demonstrates the reverse sequence. The experiments illustrate different combinations of topological defects and their trajectories during the reconfigurations, all of which can be used for novel control strategies for materials design.

We provide original explanations for the fact that F127 can promote both tangential and normal anchoring, tangential preferred at low temperature. We also note that the inner surface gives stronger tangential anchoring as a consequence of the interplay of curvature and the chemical composition and size of F127. We complement our experiments with modeling and simulations, which capture key experimental details and demonstrate the enormous complexity of the solution landscapes in nematic shells. Our modeling includes hybrid shells for the first time.

Our work is a significant step forward in the design and understanding of LC shells, and it demonstrates the power of block copolymers-so far largely ignored by the community - as LC shell stabilizers. The dynamic tunability of topology, with varying number of defects distributed in very different ways across the shell, or indeed no defects at all, opens for interesting sensors, not only of temperature but of any stimulus that affects the nematic order parameter and/or the behavior of the block copolymers at the curved LC-water interfaces. The comparatively large LC shell could act as a powerful amplifier of events taking place at the molecular scale [72], with no need for POM interrogation if selectively reflecting cholesteric LCs are used. Equally interesting is the opportunity to easily tune the programed shape change response of shell-shaped LC elastomer (LCE) actuators [38-40]. For flat LCE films there are many impres- sive demonstrations of how targeted positioning of topological defects can be used to radically change the shape shifting during actuation [73], but such control has so far been difficult to achieve in LCE shells. If block copolymer stabilizers offer the same tunability for LCE precursor materials as for cyanobiphenyls, this obstacle can be removed.

\section{ACKNOWLEDGMENTS}

We gratefully acknowledge financial support from the European Research Council under the European Union's Seventh Framework Program (FP/2007-2013)/ERC (J.P.F.L., consolidator project INTERACT, grant ID. 648763), the Fonds National de la recherche Luxembourg (J.N., grant ID 6992111 and V.S.R.J., grant ID C17/MS/11703329/trendsetter), the German Academic Exchange Service (DAAD), and the IRTG (H.-L.L.). A.M. is supported by a Leverhulme International Academic Fellowship and the University of Strathclyde's New Professors Fund. A.M. gratefully acknowledges a Visiting Professorship at the University of Bath and an OCIAM Visiting Fellowship at the University of Oxford. Y.W., A.M. and J.L. acknowledge support from ICERM in Brown University, December 2019.

\section{APPENDIX A: EXPLANATION OF APPARENT OVAL SHAPE OF TANGENTIAL-ALIGNED SHELLS}

A curious effect is that the projection of the fully tangential-aligned shells as observed in POM appears oval rather than circular, although interfacial tension certainly requires the shells to be spherical. This is an optical illusion that can be explained by the details of the director field. With the four defects located near the shell top in Fig. 6(a), $\mathbf{n}$ mainly splays outside defects 1 and 2, whereas it primarily bends outside 3 and 4 . Due to the spherical geometry of the shell, the effective birefringence $\Delta n_{\text {eff }}$ is continuously reduced towards the perimeter in the region of director splay. This is because, to an observer at the microscope, the optic axis, equivalent to $\mathbf{n}$, in this region tilts away from the sample plane, increasingly so the further we are from the center of the curved surface, hence $\Delta n_{\mathrm{eff}}$ decreases towards the perimeter. This reduction in $\Delta n_{\mathrm{eff}}$ roughly compensates for the increase towards the perimeter in the optical path length of light passing vertically through the shell. Together, the two effects result in an interference color that stays almost the same from center to edge, here in the bright yellow order of the Michel-Lévy chart.

In contrast, where $\mathbf{n}$ bends outside the 3 and 4 defects, the optic axis is oriented such that it retains its full length despite the presence of curvature, hence the full $\Delta n$ of the LC is experienced. As the increase in optical path length towards the shell perimeter now takes place at constant $\Delta n$, the effective interference moves to the right in the Michel-Lévy chart, to a more saturated pink-blue regime. The shell then appears darker, giving the false impression that its extension in the 3-4 direction is less than that in the 1-2 direction. Being aware of this feature can help to map out the director field distinctly, even without the use of a first-order $\lambda$ plate.

As is clear from the decreasing apparent overall size of the shells as they are heated from tangential via hybrid to normal configuration, the change in effective refractive index 
also affects the lensing effect that the curved LC gives rise to. Because this is further complicated by density variations with temperature, as well as the effect of disclinations, potentially affecting the thickness at different points of the shell, a complete analysis of this aspect is outside the scope of this paper.

\section{APPENDIX B: NUMERICAL METHODS}

Here we give a detailed description of our numerical methods. The experimentally observable states are modeled by the locally stable points, i.e., local or global minimizers, of the LdG free energy. We point out that there are typically multiple local energy minimizers for highly nonlinear and nonconvex problems such as the LdG minimization problem and while the global minimizer may be most frequently observed, local minimizers also have a basin of attraction and stability [65]. In particular, in an experiment, a local minimizer will not relax to the global minimizer with minimum free energy because all stable states are separated by an energy barrier.

In order to compute these locally stable points, we use spectral methods to discretize the order parameter $\mathbf{Q}$. Spectral methods are efficient numerical methods with high accuracy [74]. Several previous studies have shown that the spectral method is a powerful tool to numerical study LdG free energy [60,75-78]. The key idea to apply the spectral method to our system is to use a bispherical polar coordinate system $(\xi, \mu, \varphi)[60]$, which is given by

$$
\rho=\frac{a \sin \mu}{\cosh \xi-\cos \mu}, \quad z=\frac{a \sinh \xi}{\cosh \xi-\cos \mu}, \quad \phi=\varphi,
$$

where $(\rho, z, \phi)$ are standard cylindrical coordinates,

$$
a=\frac{1}{2 c} \sqrt{\left(1-\rho^{2}-c^{2}\right)^{2}-4 c^{2} \rho^{2}} ;
$$

for given $c$ and $\rho$. Letting $\zeta=2\left(\xi-\xi_{0}\right) /\left(\xi_{1}-\xi_{0}\right)-1$, we map the original domain to

$$
\Omega=\{(\zeta, \mu, \varphi) \mid-1 \leqslant \zeta \leqslant 1,0 \leqslant \mu<\pi, 0 \leqslant \varphi<2 \pi\} .
$$

Then, we can expand the tensor function $\mathbf{Q}(\mathbf{r})$ in terms of special functions: real spherical harmonics of $(\mu, \varphi)$ and Legendre polynomials of $\zeta$ [60]. We write

$$
\mathbf{Q}(\mathbf{r})=\sum_{l=0}^{L-1} \sum_{m=1-M}^{M-1} \sum_{n=|m|}^{N-1} \mathbf{q}_{l n m} P_{l}(\zeta) Y_{n m}(\mu, \varphi),
$$

where $L, N, M$ specify the truncation limits of the expanded series, $Y_{n m}$ is defined by

$$
Y_{n m}=P_{n}^{|m|}(\cos \mu) X_{m}(\varphi),
$$

where $P_{n}^{m}(m \geqslant 0)$ are the normalized associated Legendre polynomials and $X_{m}$ is given by

$$
X_{m}(\varphi)= \begin{cases}\cos m \varphi \quad & m \geqslant 0 \\ \sin |m| \varphi & m<0\end{cases}
$$

Due to the original symmetry of the $\mathbf{Q}$ tensor, only five elements of $\mathbf{q}_{l n m}$ are independent. Inserting (B3) into (12), we obtain a free energy as a function of these unknown tensor order parameter elements, $\mathbf{q}_{\text {lnm }}$, denoted by $F(\mathbf{q})$. The redefined free energy function is then minimized by using
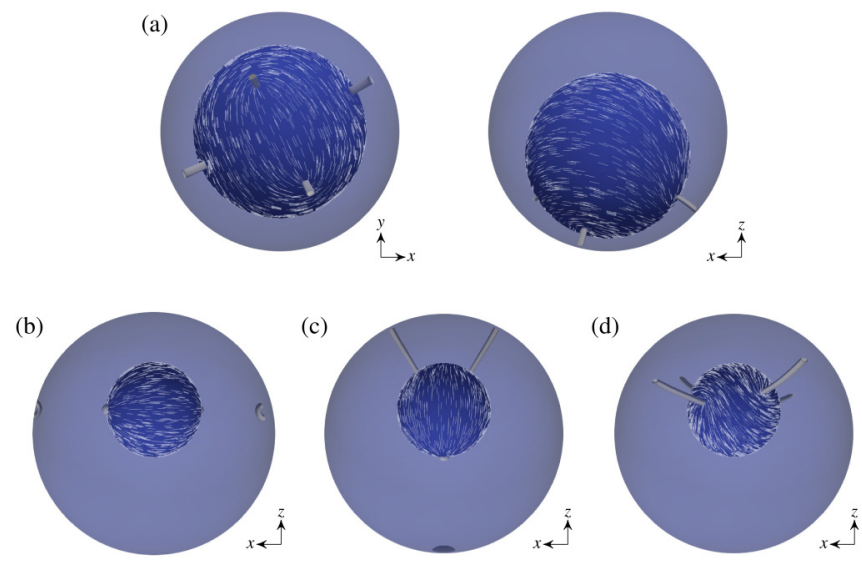

FIG. 16. Free energy minimization of thin shells in $\mathrm{T}$ configuration (a) suggests that energy minimizers have four $+1 / 2$ disclinations near the thinnest point $\left(4\left(+\frac{1}{2^{t}}\right)\right)$. The parameters are $\xi_{R}=50$, $R \approx 1 \mu \mathrm{m}, c=0.2$, and $\rho=0.7$, with $\eta=0\left(K_{1}=K_{2}=K_{3}\right)$. The shell is viewed from the thin side (left) and from the side (right), respectively. For thick shells [(b)-(d), viewed from the side; $c=0.2$ and $\rho=0.4$, all other parameters as above], all three possible defect combinations are found, $2(+1)$ in (c), $+1,2\left(+\frac{1}{2}\right)$ in (d), and $4\left(+\frac{1}{2}\right)$ in (e). We numerically capture the singular regions, drawn with tubes around each defect. In all cases, the depicted director field reflects the internal shell boundary.

a standard optimization method, such as L-BFGS [79] that treats the independent elements of tensor $\mathbf{q}_{l n m}$ as variables. Most of simulation results in this paper are obtained by taking $(N, L, M)=(64,16,64)$.

We can track the dynamics to equilibria by using the gradient flow algorithm, based on the principle that systems evolve to a local energy minimum with time or equivalently, free energy decreases with time till we settle into a local energy minimum. A general gradient flow equations can be obtained from a prescribed energy-dissipation law [80]

$$
\frac{d}{d t} \mathcal{F}[\widetilde{\mathbf{Q}}]=-\int_{\Omega} M(\boldsymbol{x}) \widetilde{\mathbf{Q}}_{t} \cdot \widetilde{\mathbf{Q}}_{t} d t,
$$

where $M(\boldsymbol{x})$ is a positive-definite matrix that determines the dynamics approaching the equilibrium, $\widetilde{\mathbf{Q}} \in \mathbb{R}^{5}$ is the vectorized order parameter $\mathbf{Q}$. By a discrete energetic variational approach [81], the gradient flow equation of $\widetilde{\mathbf{Q}}$ corresponds to energy-dissipation law (B6) can be approximated by the gradient flow equations of the coefficients $\mathbf{q}_{l n m}$, given by

$$
\frac{d}{d t} q_{l n m}^{i}=-\frac{\partial F(\mathbf{q})}{\partial q_{l n m}^{i}}, \quad i=1, \ldots, 5,
$$

with a proper choice of $M(x)$. We can solve (B7) by an explicit Euler method with adaptive stepsize, which updates the $q_{l n m}^{i}$ 's according to

$$
\left(q_{\text {lnm }}^{i}\right)^{n+1}=\left(q_{\text {lnm }}^{i}\right)^{n}-\left.\epsilon_{n} \frac{\partial F(\mathbf{q})}{\partial q_{\text {lnm }}^{i}}\right|_{\mathbf{q}=\mathbf{q}^{n}},
$$

where $\epsilon_{n}$ is the step length for $n$th iteration. We choose $\epsilon_{n}$ as Barzilai-Borwein step size [82]. We use the gradient flow algorithm to mimic transitions from fully tangential shells to hybrid shells and vice versa in what follows. 


\section{APPENDIX C: SIMULATED FULLY TANGENTIAL SHELLS}

To benchmark our numerics with respect to previous simulations, comparing also with our experimental results, we simulate $\mathrm{T}$ configurations using the one-constant approximation, i.e., $\eta=0$. For thin shells, only the T3 $\left[4\left(+1 / 2^{t}\right)\right]$ configuration is found by direct minimization, see Fig. 16(a). For thicker shells, we can find three equilibrium $\mathrm{T}$ configurations also in simulation, with defects no longer concentrated to the thinnest point: a $+1^{l},+1^{r}$, where $l$ stands for "left" and $r$ for "right" [Fig. 16(b)], a $+1^{b}, 2\left(+1 / 2^{t}\right)$ [Fig. 16(c)], and a 4(+1/2t arrangement [Fig. 16(d)]. The simulation results are largely compatible with previous reports on simulations of shells in $\mathrm{T}$ configuration $[9,44-46]$.
[1] M. Urbanski, C. G. Reyes, J. Noh, A. Sharma, Y. Geng, V. S. R. Jampani, and J. P. Lagerwall, J. Phys.: Condens. Matter 29, 133003 (2017).

[2] J. P. F. Lagerwall and G. Scalia, Curr. Appl. Phys. 12, 1387 (2012).

[3] T. Lopez-Leon and A. Fernandez-Nieves, Colloid Polym. Sci. 289, 345 (2011).

[4] D. R. Nelson, Nano. Lett. 2, 1125 (2002).

[5] A. Fernandez-Nieves, V. Vitelli, A. S. Utada, D. R. Link, M. Marquez, D. R. Nelson, and D. A. Weitz, Phys. Rev. Lett. 99, 157801 (2007).

[6] G. Skacej and C. Zannoni, Phys. Rev. Lett. 100, 197802 (2008).

[7] T. Lopez-Leon and A. Fernandez-Nieves, Phys. Rev. E 79, 021707 (2009).

[8] S. Kralj, R. Rosso, and E. G. Virga, Soft Matter 7, 670 (2011).

[9] T. Lopez-Leon, V. Koning, K. B. S. Devaiah, V. Vitelli, and A. Fernandez-Nieves, Nat. Phys. 7, 391 (2011).

[10] G. Napoli and L. Vergori, Phys. Rev. E 85, 061701 (2012).

[11] G. Napoli and L. Vergori, Phys. Rev. Lett. 108, 207803 (2012).

[12] H.-L. Liang, E. Enz, G. Scalia, and J. Lagerwall, Mol. Cryst. Liq. Cryst. 549, 69 (2011).

[13] H.-L. Liang, S. Schymura, P. Rudquist, and J. Lagerwall, Phys. Rev. Lett. 106, 247801 (2011).

[14] T. Lopez-Leon, A. Fernandez-Nieves, M. Nobili, and C. Blanc, Phys. Rev. Lett. 106, 247802 (2011).

[15] H.-L. Liang, R. Zentel, P. Rudquist, and J. Lagerwall, Soft Matter 8, 5443 (2012).

[16] T. Lopez-Leon, A. Fernandez-Nieves, M. Nobili, and C. Blanc, J. Phys.-Condens. Matter. 24, 284122 (2012).

[17] H. Liang, J. Noh, R. Zentel, P. Rudquist, and J. Lagerwall, Philos. Transact. A Math. Phys. Eng. Sci. 371, 20120258 (2013).

[18] O. Manyuhina and M. Bowick, Int. J. Non-Linear Mech. 75, 87 (2015).

[19] T. Akita, H. Kouno, Y. Iwai, Y. Uchida, and N. Nishiyama, J. Mater. Chem. C 5, 1303 (2017).

[20] Y. Uchida, Y. Takanishi, and J. Yamamoto, Adv. Mater. 25, 3234 (2013).

[21] Y. Geng, J.-H. Jang, K.-G. Noh, J. Noh, J. P. Lagerwall, and S.-Y. Park, Adv. Opt. Mater. 6, 1700923 (2018).

[22] Y. Geng, J. Noh, I. Drevensek-olenik, R. Rupp, and J. P. F. Lagerwall, Liq. Cryst. 44, 1948 (2017).

[23] L. Tran, M. O. Lavrentovich, G. Durey, A. Darmon, M. F. Haase, N. Li, D. Lee, K. J. Stebe, R. D. Kamien, and T. Lopez-Leon, Phys. Rev. X 7, 041029 (2017).

[24] A. Darmon, M. Benzaquen, D. Seč, S. Čopar, O. Dauchot, and T. Lopez-Leon, Proc. Natl. Acad. Sci. USA 113, 9469 (2016).
[25] A. Darmon, M. Benzaquen, S. Copar, O. Dauchot, and T. Lopez-Leon, Soft Matter 12, 9280 (2016).

[26] Y. Geng, J. Noh, I. Drevensek-Olenik, R. Rupp, G. Lenzini, and J. P. F. Lagerwall, Sci. Rep. 6, 26840 (2016).

[27] S. Lee, H. Seo, Y. Kim, and S. Kim, Adv. Mater. 29, 1606894 (2017).

[28] I.-S. Heo and S.-Y. Park, Sens. Actuators, B 251, 658 (2017).

[29] J. Noh, B. Henx, and J. P. Lagerwall, Adv. Mater. 28, 10170 (2016).

[30] Y. Iwai, R. Iijima, K. Yamamoto, T. Akita, Y. Uchida, and N. Nishiyama, Adv. Opt. Mater. 8, 1901363 (2020).

[31] D. Myung and S. Park, ACS Appl Mater Interfaces 11, 20350 (2019).

[32] M. Schwartz, G. Lenzini, Y. Geng, P. Rønne, P. Ryan, and J. Lagerwall, Adv. Mater. 30, 1707382 (2018).

[33] G. Lenzini, S. Ouchani, P. Roenne, P. Y. Ryan, Y. Geng, J. Lagerwall, and J. Noh, IEEE Workshop on Information Forensics and Security (IEEE, Rennes, France, 2017).

[34] J.-G. Kim and S.-Y. Park, Adv. Opt. Mater. 5, 1700243 (2017).

[35] J. Kang, S. Kim, A. Fernandez-Nieves, and E. Reichmanis, J. Am. Chem. Soc. 139, 5708 (2017).

[36] A. Sharma, V. Jampani, and J. Lagerwall, Langmuir 35, 11132 (2019).

[37] J.-H. Jang and S.-Y. Park, Sens. Actuators, B 241, 636 (2017).

[38] V. S. R. Jampani, R. H. Volpe, K. Reguengo de Sousa, J. Ferreira Machado, C. M. Yakacki, and J. P. F. Lagerwall, Sci. Adv. 5, eaaw2476 (2019).

[39] V. S. R. Jampani, D. J. Mulder, K. R. De Sousa, A.-H. Gélébart, J. P. F. Lagerwall, and A. P. H. J. Schenning, Adv. Funct. Mater. 28, 1801209 (2018).

[40] E.-K. Fleischmann, H.-L. Liang, N. Kapernaum, F. Giesselmann, J. P. F. Lagerwall, and R. Zentel, Nat. Commun. 3, 1178 (2012).

[41] A. Sharma and J. P. F. Lagerwall, Liq. Cryst. 45, 2319 (2018).

[42] J. Noh, K. Reguengo De Sousa, and J. P. F. Lagerwall, Soft Matter 12, 367 (2016).

[43] G. Volovik and O. Lavrentovich, Zh. Eksp. Teor. Fiz. 85, 1159 (1983).

[44] D. Seč, T. Lopez-Leon, M. Nobili, C. Blanc, A. FernandezNieves, M. Ravnik, and S. Žumer, Phys. Rev. E 86, 020705(R) (2012).

[45] V. Koning, T. Lopez-Leon, A. Darmon, A. Fernandez-Nieves, and V. Vitelli, Phys. Rev. E 94, 012703 (2016).

[46] V. Koning, T. Lopez-Leon, A. Fernandez-Nieves, and V. Vitelli, Soft Matter 9, 4993 (2013).

[47] J. Noh, V. S. R. Jampani, O. Haba, K. Yonetake, H. Takezoe, and J. P. Lagerwall, J. Mol. Liq. 267, 197 (2018).

[48] I. I. Smalyukh, S. Shiyanovskii, and O. Lavrentovich, Chem. Phys. Lett. 336, 88 (2001). 
[49] P. G. de Gennes and J. Prost, The Physics of Liquid Crystals (Oxford University Press, Oxford, UK, 1993), Vol. 83.

[50] A. Majumdar, Eur. J. Appl. Math. 21, 181 (2010).

[51] A. Majumdar and Y. Wang, J. Math. Anal. Appl. 464, 328 (2018).

[52] E. G. Virga, Variational Theories for Liquid Crystals (CRC Press, 1995), Vol. 8.

[53] N. J. Mottram and C. Newton, University of Strathclyde, Department of Mathematics research report 2004, 10 (2004).

[54] J. M. Ball and A. Majumdar, Mol. Cryst. Liq. Cryst. 525, 1 (2010).

[55] R. Williams, J. Phys. A: Math. Gen. 19, 3211 (1986).

[56] C. Luo, A. Majumdar, and R. Erban, Phys. Rev. E 85, 061702 (2012).

[57] J.-B. Fournier and P. Galatola, EPL (Europhysics Letters) 72, 403 (2005).

[58] I. Muševič, M. Škarabot, U. Tkalec, M. Ravnik, and S. Žumer, Science 313, 954 (2006).

[59] M. Ravnik and S. Žumer, Liq. Cryst. 36, 1201 (2009).

[60] Y. Wang, P. Zhang, and J. Z. Y. Chen, Phys. Rev. E 96, 042702 (2017).

[61] M. A. Gharbi, D. Seč, T. Lopez-Leon, M. Nobili, M. Ravnik, S. Žumer, and C. Blanc, Soft Matter 9, 6911 (2013).

[62] M. Sadati, Y. Zhou, D. Melchert, A. Guo, J. A. MartinezGonzalez, T. F. Roberts, R. Zhang, and J. J. de Pablo, Soft Matter 13, 7465 (2017).

[63] See Supplemental Material at http://link.aps.org/supplemental/ 10.1103/PhysRevResearch.2.033160 for movies showing F127stabilized 5CB shells on heating/cooling (M1), F127-stabilized E7 shells on heating from different start configurations (M2M4), E7 shells stabilized by F127 and SDS on heating (M5M6), FCPM visualization of F127-stabilized hybrid E7 shell (M7), and reference experiment with PVA-stabilized E7 shells (M8).
[64] P. Alexandridis, J. F. Holzwarth, and T. A. Hatton, Macromolecules 27, 2414 (1994).

[65] J. Yin, Y. Wang, J. Z. Y. Chen, P. Zhang, and L. Zhang, Phys. Rev. Lett. 124, 090601 (2020).

[66] P. Linse, J. Phys. Chem. 97, 13896 (1993).

[67] P. Linse, Macromolecules 26, 4437 (1993).

[68] J. Brake and N. Abbott, Langmuir 18, 6101 (2002).

[69] G. Durey and T. Lopez-Leon, arXiv:1811.09119v1.

[70] S.-D. Kim, B. Lee, S.-W. Kang, and J.-K. Song, Nat. Commun. 6, 7936 (2015).

[71] O. Lavrentovich and V. Sergan, Il Nuovo Cimento D 12, 1219 (1990).

[72] R. J. Carlton, J. T. Hunter, D. S. Miller, R. Abbasi, P. C. Mushenheim, L. N. Tan, and N. L. Abbott, Liq. Cryst. Rev. 1, 29 (2013).

[73] T. White and D. Broer, Nat. Mater. 14, 1087 (2015).

[74] J. Shen, T. Tang, and L.-L. Wang, Spectral Methods: Algorithms, Analysis and Applications (Springer Science \& Business Media, 2011), Vol. 41.

[75] Y. Hu, Y. Qu, and P. Zhang, Commun. Comput. Phys. 19, 354 (2016).

[76] Y. Tong, Y. Wang, and P. Zhang, Numer. Math. 10, 205 (2017).

[77] Y. Wang, P. Zhang, and J. Z. Chen, Soft Matter 14, 6756 (2018).

[78] G. Canevari, J. Harris, A. Majumdar, and Y. Wang, Int. J. NonLinear Mech. 119, 103342 (2020).

[79] S. Wright and J. Nocedal, Numerical Optimization (Springer, 1999), Vol. 35.

[80] M.-H. Giga, A. Kirshtein, and C. Liu, in Handbook of Mathematical Analysis in Mechanics of Viscous Fluids, edited by Y. Giga and A. Novotny (Springer International, 2017), pp. 1-41.

[81] C. Liu and Y. Wang, J. Comput. Phys. 417, 109566 (2020).

[82] J. Barzilai and J. M. Borwein, IMA J. Numer. Anal. 8, 141 (1988). 